\title{
Meshfree co-rotational formulation for two-dimensional continua
}

\author{
L. L. Yaw, N. Sukumar*, S. K. Kunnath \\ Department of Civil and Environmental Engineering, University of California, Davis, CA 95616, USA
}

\section{SUMMARY}

In this paper, a meshfree co-rotational formulation for two-dimensional continua is proposed. In a co-rotational formulation, the motion of a body is separated into rigid motion and strain producing deformation. Traditionally, this has been done in the setting of finite elements for beams and shell type elements. In the present work every node in a meshfree discretized domain has its own corotating coordinate system. Three key ingredients are established in order to apply the co-rotational formulation: (i) the relationship between global and local variables, (ii) the angle of rotation of a typical co-rotating coordinate system, and (iii) a variationally consistent tangent stiffness matrix. An algorithm for the co-rotational formulation based on load control is provided. Maximum-entropy basis functions are used to discretize the domain and stabilized nodal integration is implemented to construct the global system of equations. Numerical examples are presented to demonstrate the validity of the meshfree co-rotational formulation. Copyright (c) 2008 John Wiley \& Sons, Ltd.

KEY WORDS: co-rotational, nodal integration, stabilization, meshfree method, maximum-entropy, convex approximation

\footnotetext{
${ }^{*}$ Correspondence to: N. Sukumar, Department of Civil and Environmental Engineering, University of California, One Shields Avenue, Davis, CA 95616, USA. E-mail: nsukumar@ucdavis.edu

Contract/grant sponsor: National Science Foundation; contract/grant number: CMMI-0826513,CMMI-0626481
} 


\section{INTRODUCTION}

Co-rotational formulations are commonly used in finite element formulations for the analysis of structures. Wempner [1] and Belytschko and Hsieh [2] pioneered the introduction of corotational formulations in finite element analysis. Such a formulation has many commonalities with the 'natural approach' of Argyris et al. [3]. The co-rotational formulation is very popular for beams and shell elements and it has been extended to include finite strains with continuum elements in a consistent formulation by Crisfield and Moita $[4,5]$. One of the primary motivations of a co-rotational formulation is the ability to use linear elements in a non-linear context; see, for example, the work of Felippa and Haugen [6]. Thus far, the co-rotational formulation has only been implemented using finite elements. In this paper, meshfree basis functions are introduced within the framework of a co-rotational formulation for continua. To the authors' knowledge this has not been previously introduced in the literature.

This work is part of a larger effort to develop a new computational framework for collapse analysis of structures. This framework attempts to take advantage of the finite element (FE) method where meshfree is not an efficient option and to utilize meshfree methods where $\mathrm{FE}$ is not viable. Collapse simulation is by its nature a problem that is highly nonlinear, involving large displacements, rotations and inelastic material behavior. While finite-element based simulations of structural collapse and failure have met with some success for limited applications [7-9], much of the effort using finite elements to simulate large displacements have encountered numerous difficulties due to mesh distortions which cause a need for remeshing, loss of accuracy, and at times unsuccessful completion of the simulation altogether. These 
difficulties are observed for both continuum elements as well as beam elements. Significant work has gone into the development of beam elements for limit state analysis of large-scale engineering structures. However, as noted by Torkamani [10], these methods tend to have two principal deficiencies: inaccurate descriptions of material nonlinearity, and an inability to properly capture large distortions across the length of the element. Fiber-based beam elements have been used to improve modeling of material nonlinearity [10-12] and Lagrangian or corotational formulations are employed to include large deflections. Despite these advances, the ability to simulate collapse is still inadequate.

The objective of this work, therefore, is to explore a new paradigm for nonlinear structural analysis computation by examining the ability of meshfree methods to alleviate some of these difficulties. Since collapse of a structural component is typically a result of large member rotations at beam-column joint interfaces, it is perceived that the advantages of meshfree methods can be combined with those of a co-rotational formulation without the need to resort to finite strains, thereby enabling the simulation of large displacements associated with collapse conditions. Furthermore, by using a continuum approach, material behavior is modeled more accurately through the cross-section of beam-type structural elements. As an essential first step toward the eventual goal of developing a FE-meshfree framework for large-deformation analysis of structural systems to aid in collapse simulations, the present paper focuses on twodimensional continua in the presence of small strains with elastic and elasto-plastic material behavior.

The remainder of this paper is organized as follows. In Section 2, the co-rotational formulation is derived to give (i) the relationship between global and local variables, (ii) the angle of rotation of a typical co-rotating coordinate system, and (iii) a variationally Copyright (C) 2008 John Wiley \& Sons, Ltd.

Int. J. Numer. Meth. Engng 2008; 00:1-38

Prepared using nmeauth.cls 
consistent tangent stiffness matrix. Including inelastic material behavior is also discussed followed by an algorithm for a co-rotational formulation in a meshfree setting. In Section 3, the derivation of maximum-entropy (max-ent) basis functions is presented along with details on the implementation of nodal integration and stabilization. Section 4 presents numerical examples for validation of the proposed formulation, which is followed by some concluding remarks in Section 5.

\section{CO-ROTATIONAL FORMULATION}

In general, the motion of a body is composed of rigid body translation, rigid body rotation and strain producing deformations. Consider a sufficiently small region $\Omega \subset \mathbb{R}^{2}$ of a body. To this small region, attach a local coordinate frame that rotates and translates with the material points of the region. With respect to this local coordinate frame, the rigid body rotations and translations, of the small region's overall motion, are negligible and only local strainproducing deformations remain. This is the key idea behind a co-rotational formulation. It is the objective of a co-rotational formulation to perform a nonlinear analysis of a structure and determine the global displacement behavior as well as the stress and strain causing local deformations. Some of the advantages of a co-rotational formulation are as follows. First, for small strain/large rotation problems, Mattiasson [13] indicates that the co-rotational formulation is more accurate and has better convergence properties than finite strain total Lagrangian or updated Lagrangian formulations. Second, co-rotational formulations satisfy the principle of material frame indifference [14]. As a result of material frame invariance, damage constitutive equations are not limited to isotropic elastic response [15]. Third, inelastic type constitutive equations take the same form as in the case of a small deformation theory Copyright (C) 2008 John Wiley \& Sons, Ltd.

Int. J. Numer. Meth. Engng 2008; 00:1-38

Prepared using nmeauth.cls 
since stresses and strain tensors are objective [16]. This greatly simplifies integration of inelastic constitutive equations. Lastly, geometric nonlinearities due to large displacements and rotations are taken into account without the requirement of a finite strain formulation and alternative stress definitions.

For a co-rotational formulation several key ingredients are necessary, namely (i) the relationship between global and local variables, (ii) a method for determining the angle of rotation of a typical co-rotating coordinate system, and (iii) the expression for a variationally consistent tangent stiffness matrix. These ingredients are described within the following sections, where for the sake of clarity and completeness, intermediate steps in the derivation are also indicated. The ensuing presentation closely follows Crisfield and Moita [4].

\subsection{Relationship between global and local variables}

Referring to Figure 1, the relationship between overall global deformations and the local strain producing deformations is illustrated. In Figure 1, node $L$ and its neighboring nodes are shown. In general, there are $n$ nodes (node $L$ is included in the set of $n$ nodes) to which a local corotating coordinate frame is associated (for finite elements the coordinate frame is usually attached to each element). For simplicity only four nodes are shown and the local co-rotating frame origin is placed at node $L$. In the reference configuration, the local co-rotating frame axes are parallel to the global axes. Due to displacement of the overall structure the $n$ nodes translate, rotate and deform to some current configuration as shown.

From Figure 1, the local nodal coefficients (nodal displacements for the case of finite elements) for node $i$ in the local coordinate frame are expressed as

$$
\mathbf{d}_{\ell}^{i}=\mathbf{Q}^{T} \mathbf{x}^{i L}-\mathbf{X}_{\ell}^{i}
$$

Copyright (C) 2008 John Wiley \& Sons, Ltd.

Int. J. Numer. Meth. Engng 2008; 00:1-38

Prepared using nmeauth.cls 


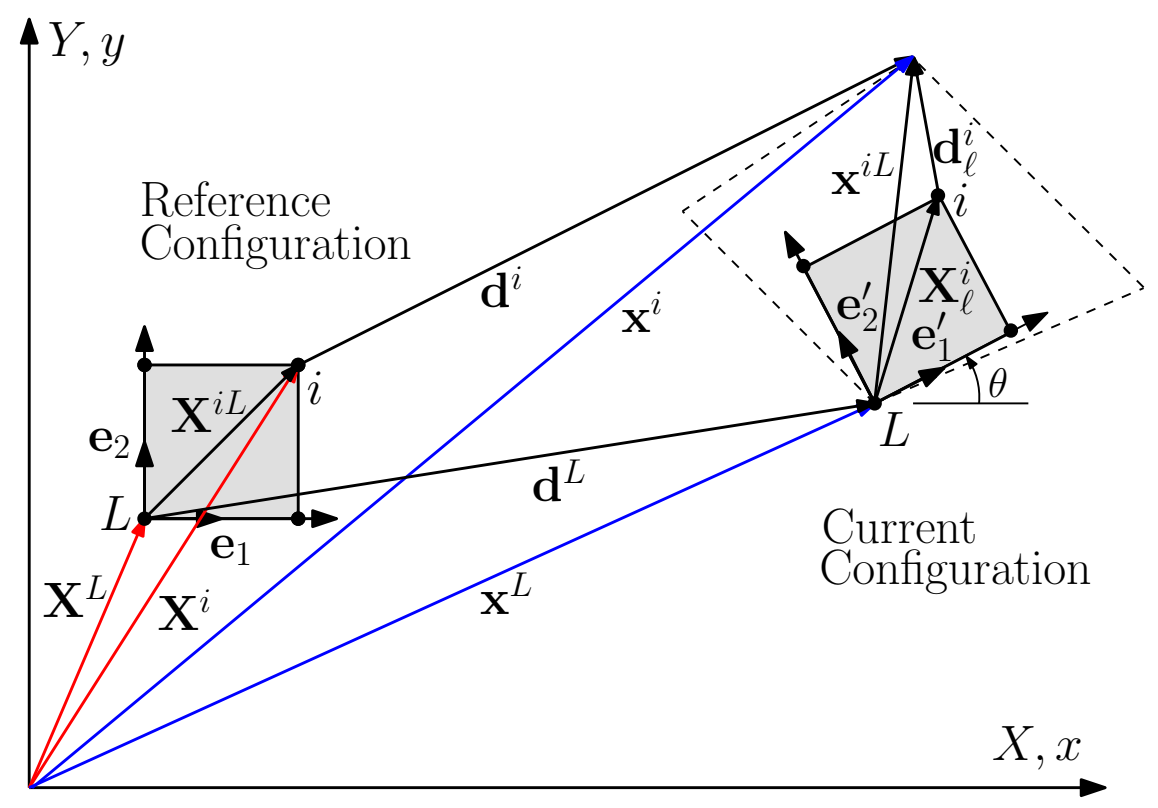

Figure 1. Reference and current configurations in co-rotational formulation.

where a subscript $\ell$ is attached to vectors with components in local coordinates (with some exceptions such as stress, $\boldsymbol{\sigma}$, and strain-displacement matrices, $\mathbf{B}$, which are understood to be in the local coordinates of a co-rotational formulation) and $\mathbf{x}^{i L}=\mathbf{x}^{i}-\mathbf{x}^{L}=\mathbf{X}^{i L}+\mathbf{d}^{i}-\mathbf{d}^{L}$ indicates the difference between the spatial coordinates of nodes $i$ and $L$ in the current configuration with components in the global coordinate system. The orthogonal matrix $\mathbf{Q}=\left[\mathbf{e}_{1}^{\prime} \mathbf{e}_{2}^{\prime}\right]$ is a rotation matrix, so that $\mathbf{Q}^{T}$ transforms global vector components to local vector components. The unit basis vectors $\mathbf{e}_{1}^{\prime}$ and $\mathbf{e}_{2}^{\prime}$ which define the local co-rotating coordinate frame are defined in terms of $\theta$ with global components as follows:

$$
\mathbf{e}_{1}^{\prime}=\left[\begin{array}{c}
\cos \theta \\
\sin \theta
\end{array}\right] \quad \mathbf{e}_{2}^{\prime}=\left[\begin{array}{c}
-\sin \theta \\
\cos \theta
\end{array}\right] .
$$

Lastly, $\mathbf{X}_{\ell}^{i}$ represents the material coordinates of node $i$ in the local coordinate frame. It is Copyright (C) 2008 John Wiley \& Sons, Ltd.

Int. J. Numer. Meth. Engng 2008; 00:1-38

Prepared using nmeauth.cls 
noted that $\mathbf{X}_{\ell}^{i}=\mathbf{Q}^{T} \mathbf{X}^{i L}=\mathbf{Q}^{T}\left(\mathbf{X}^{i}-\mathbf{X}^{L}\right)$.

Based on the reference and current configurations, (1) expresses the local nodal coefficient components for node $i$ in terms of known quantities $\mathbf{x}^{i L}, \mathbf{X}_{\ell}^{i}$ and as yet unknown quantity $\theta$, the angle of rotation of the local co-rotating coordinate frame. This unknown quantity is determined in the next section.

\subsection{Co-rotating frame angle of rotation}

The angle of rotation of the co-rotated coordinate frame is found by assuming that the local spin, due to local nodal displacements in the current configuration, is equal to zero (see Jetteur and Cescotto [17]). The local spin is evaluated at the centroid of the Voronoi cell for node $L$ in the reference configuration by making use of the following equation, which is a special case of the polar decomposition theorem in two-dimensions:

$$
\Omega_{\ell}=\frac{\partial u_{1 \ell}}{\partial Y_{\ell}}-\frac{\partial u_{2 \ell}}{\partial X_{\ell}}=0
$$

The meshfree approximation for the displacement field in terms of the local nodal coefficients, $\mathbf{d}_{\ell}$, is written as

$$
u_{j \ell}=\phi^{T} \mathbf{d}_{j \ell}, \quad(j=1,2)
$$

where $\phi$ is the vector of nodal basis functions and $\mathbf{d}_{j \ell}$ denotes the vector of local nodal coefficients associated with degree of freedom $j$.

Substituting (4) into (3) gives

$$
\Omega_{\ell}=\left(\frac{\partial \phi}{\partial Y_{\ell}}\right)^{T} \mathbf{d}_{1 \ell}-\left(\frac{\partial \phi}{\partial X_{\ell}}\right)^{T} \mathbf{d}_{2 \ell}=\mathbf{a}_{\ell}^{T} \mathbf{d}_{\ell}
$$

Copyright (C) 2008 John Wiley \& Sons, Ltd.

Int. J. Numer. Meth. Engng 2008; 00:1-38

Prepared using nmeauth.cls 
where

$$
\mathbf{a}_{\ell}=\left[\begin{array}{c}
\frac{\partial \phi_{1}}{\partial Y_{\ell}} \\
-\frac{\partial \phi_{1}}{\partial X_{\ell}} \\
\vdots \\
\frac{\partial \phi_{n}}{\partial Y_{\ell}} \\
-\frac{\partial \phi_{n}}{\partial X_{\ell}}
\end{array}\right] \quad \text { and } \quad \mathbf{d}_{\ell}=\left[\begin{array}{c}
d_{1 \ell}^{1} \\
d_{2 \ell}^{1} \\
d_{1 \ell}^{2} \\
d_{2 \ell}^{2} \\
\vdots \\
d_{1 \ell}^{n} \\
d_{2 \ell}^{n}
\end{array}\right]
$$

Note that $\mathbf{a}_{\ell}$ is evaluated at the centroid of the Voronoi cell for node $L$ in local material coordinates $\mathbf{X}_{\ell}$ (which is equivalent to evaluation in global material coordinates $\mathbf{X}$ ) and hence is a fixed vector. Next substitute (1) into (5) to get

$$
\Omega_{\ell}=\sum\left(\mathbf{a}_{\ell}^{i}\right)^{T}\left(\mathbf{Q}^{T} \mathbf{x}^{i L}\right)-\sum\left(\mathbf{a}_{\ell}^{i}\right)^{T}\left(\mathbf{X}_{\ell}^{i}\right)=0 .
$$

Noting that the last term of (6) is zero and expanding the first term yields

$$
\Omega_{\ell}=\sum\left(\mathbf{a}_{\ell}^{i}\right)^{T}\left(\cos \theta\left(\begin{array}{c}
x^{i L} \\
y^{i L}
\end{array}\right)+\sin \theta\left(\begin{array}{c}
y^{i L} \\
-x^{i L}
\end{array}\right)\right)=0,
$$

or

$$
\Omega_{\ell}=a \sin \theta+b \cos \theta=0, \quad a=\sum\left(\mathbf{a}_{\ell}^{i}\right)^{T}\left(\begin{array}{c}
y^{i L} \\
-x^{i L}
\end{array}\right), \quad b=\sum\left(\mathbf{a}_{\ell}^{i}\right)^{T}\left(\begin{array}{c}
x^{i L} \\
y^{i L}
\end{array}\right)
$$

The relationships in (7) are more conveniently expressed as:

$$
a=\mathbf{c}^{T} \overline{\mathbf{x}}
$$

Copyright (C) 2008 John Wiley \& Sons, Ltd.

Int. J. Numer. Meth. Engng 2008; 00:1-38

Prepared using nmeauth.cls 
where

$$
\mathbf{c}=\left[\begin{array}{ccccccc}
0 & -1 & 0 & 0 & \ldots & 0 & 0 \\
1 & 0 & 0 & 0 & & 0 & 0 \\
0 & 0 & 0 & -1 & & \vdots \\
0 & 0 & 1 & 0 & & & \\
\vdots & & & & \ddots & & \\
0 & 0 & & & & 0 & -1 \\
0 & 0 & \ldots & & & 1 & 0
\end{array}\right] \mathbf{a} \ell, \quad \overline{\mathbf{x}}=\left[\begin{array}{c}
x^{1 L} \\
y^{1 L} \\
x^{2 L} \\
y^{2 L} \\
\vdots \\
x^{n L} \\
y^{n L}
\end{array}\right]
$$

and $b=\mathbf{a}_{\ell}^{T} \overline{\mathbf{x}}$. Note that $\mathbf{c}$ in (9) is a $2 n$ by $2 n$ matrix depending on the number of neighbors $n$ and similarly $\overline{\mathbf{x}}$ is a $2 n$ by 1 vector. With these expressions in hand it is possible to solve for the angle of rotation $\theta$, which from (7) is

$$
\theta=\tan ^{-1}\left(\frac{-b}{a}\right)
$$

\subsection{Derivation of the tangent stiffness matrix}

To derive the tangent stiffness matrix first consider the local internal force vector, $\mathbf{q}_{L \ell}$, for node $L$ and its neighboring nodes, which is written as

$$
\mathbf{q}_{L \ell}=\int_{\Omega} \mathbf{B}^{T} \boldsymbol{\sigma} d V=\mathbf{K}_{\ell} \mathbf{d}_{\ell}
$$

where $\mathbf{B}$ is the local strain-displacement matrix, $\boldsymbol{\sigma}$ are the local Cauchy stresses and $\mathbf{K}_{\ell}$ represents the local stiffness matrix.

Next, note that the local nodal coefficients, $\mathbf{d}_{\ell}$, are related to the global nodal coefficients, d, via some function, $f$, i.e.,

$$
\mathbf{d}_{\ell}=f\left(\mathbf{d}, \mathbf{e}_{1}^{\prime}, \mathbf{e}_{2}^{\prime}\right),
$$


and the variation of (12) leads to the relationship

$$
\delta \mathbf{d}_{\ell}=\mathbf{T} \delta \mathbf{d}
$$

where $\mathbf{T}$ is some as yet to be determined transformation matrix. Virtual work at the local and global level are equivalent so that

$$
\left(\delta \mathbf{d}_{\ell}\right)^{T} \mathbf{q}_{L \ell}=(\delta \mathbf{d})^{T} \mathbf{q}_{L} .
$$

The global internal forces in terms of the local internal forces are found by making use of (11), (13) and (14), which yields

$$
\mathbf{q}_{L}=\mathbf{T}^{T} \mathbf{K}_{\ell} \mathbf{d}_{\ell}
$$

To obtain the global stiffness matrix the variation of (15) is taken, which gives

$$
\delta \mathbf{q}_{L}=\mathbf{T}^{T} \delta \mathbf{q}_{L \ell}+\delta \mathbf{T}^{T} \mathbf{q}_{L \ell}=\mathbf{T}^{T} \mathbf{K}_{t \ell} \delta \mathbf{d}_{\ell}+\mathbf{K}_{t \sigma} \delta \mathbf{d}=\mathbf{T}^{T} \mathbf{K}_{t \ell} \mathbf{T} \delta \mathbf{d}+\mathbf{K}_{t \sigma} \delta \mathbf{d}
$$

where $\delta \mathbf{T}^{T} \mathbf{q}_{L \ell}$ is represented as shown by $\mathbf{K}_{t \sigma} \delta \mathbf{d}$. The matrix $\mathbf{K}_{t \sigma}$ is the initial stiffness matrix, $\mathbf{K}_{t \ell}$ is the local tangent stiffness matrix (possibly constructed by considering inelastic material behavior) equal to $\mathbf{K}_{\ell}$ for small strains and linear elasticity and the last equality in (16) is found by making use of (13). Equation (16) yields

$$
\delta \mathbf{q}_{L}=\left[\mathbf{T}^{T} \mathbf{K}_{t \ell} \mathbf{T}+\mathbf{K}_{t \sigma}\right] \delta \mathbf{d}=\mathbf{K}_{T} \delta \mathbf{d},
$$

where $\mathbf{K}_{T}$ represents the tangent stiffness matrix at the global level.

To find the transformation matrix in (17), the variation of (1) is taken to give

$$
\delta \mathbf{d}_{\ell}^{i}=\mathbf{Q}^{T} \delta \mathbf{x}^{i L}+\delta \mathbf{Q}^{T} \mathbf{x}^{i L} .
$$

From Figure 1, note that

$$
\mathbf{x}^{i L}=\mathbf{X}^{i L}+\mathbf{d}^{i}-\mathbf{d}^{L}=\mathbf{X}^{i L}+\mathbf{d}^{i L} .
$$


Taking the variation of (19) gives

$$
\delta \mathbf{x}^{i L}=\delta \mathbf{X}^{i L}+\delta \mathbf{d}^{i L}=\delta \mathbf{d}^{i L},
$$

where the last step results since $\delta \mathbf{X}^{i L}$ is zero. Substituting (20) into (18) yields

$$
\delta \mathbf{d}_{\ell}^{i}=\mathbf{Q}^{T} \delta \mathbf{d}^{i L}+\delta \mathbf{Q}^{T} \mathbf{x}^{i L}
$$

Taking the variation of $\mathbf{Q}^{T}$ gives

$$
\delta \mathbf{Q}^{T}=\delta\left[\begin{array}{ll}
\mathbf{e}_{1}^{\prime} & \mathbf{e}_{2}^{\prime}
\end{array}\right]^{T}=\left[\begin{array}{cc}
-\sin \theta & -\cos \theta \\
\cos \theta & -\sin \theta
\end{array}\right]^{T} \delta \theta .
$$

Consequently,

$$
\delta \mathbf{Q}^{T} \mathbf{x}^{i L}=\left[\begin{array}{cc}
-s & c \\
-c & -s
\end{array}\right]\left(\begin{array}{c}
x^{i L} \\
y^{i L}
\end{array}\right) \delta \theta=\left[\begin{array}{c}
-s x^{i L}+c y^{i L} \\
-c x^{i L}-s y^{i L}
\end{array}\right] \delta \theta=\mathbf{Q}^{T}\left(\begin{array}{c}
y^{i L} \\
-x^{i L}
\end{array}\right) \delta \theta .
$$

Now substituting (22) into (21) yields

$$
\delta \mathbf{d}_{\ell}^{i}=\mathbf{Q}^{T} \delta \mathbf{d}^{i L}+\mathbf{Q}^{T}\left(\begin{array}{c}
y^{i L} \\
-x^{i L}
\end{array}\right) \delta \theta .
$$

If $\mathbf{Q}^{T} \delta \mathbf{d}^{L}$ is added to (23) it should have no effect if the local coordinate system computations correctly satisfy the infinitesimal strain-free rigid body requirements (when extended to finite strains the reader is referred to the work of Rankin [18], where this assumption is avoided). This addition to (23) gives

$$
\delta \mathbf{d}_{\ell}^{i}=\mathbf{Q}^{T} \delta \mathbf{d}^{i}+\mathbf{Q}^{T}\left(\begin{array}{c}
y^{i L} \\
-x^{i L}
\end{array}\right) \delta \theta .
$$

To obtain $\delta \theta$, differentiate (10) by recalling that $\frac{d\left(\tan ^{-1} u\right)}{d x}=\frac{1}{1+u^{2}} \frac{d u}{d x}$. This gives

$$
\delta \theta=\frac{1}{1+\frac{b^{2}}{a^{2}}} \delta\left(-b a^{-1}\right)=\frac{a^{2}}{a^{2}+b^{2}}\left(-\delta b a^{-1}+a^{-2} b \delta a\right)=\frac{a^{2}}{a^{2}+b^{2}}\left(\frac{b \delta a}{a^{2}}-\frac{a \delta b}{a^{2}}\right) .
$$

Copyright (C) 2008 John Wiley \& Sons, Ltd.

Int. J. Numer. Meth. Engng 2008; 00:1-38

Prepared using nmeauth.cls 
Rearranging and simplifying (25) yields

$$
\delta \theta=\frac{b \delta a-a \delta b}{a^{2}+b^{2}}=\frac{1}{a^{2}+b^{2}}\left(b \mathbf{c}^{T}-a \mathbf{a}_{\ell}^{T}\right) \delta \mathbf{d}=\mathbf{v}^{T} \delta \mathbf{d} .
$$

Substituting $\delta \theta=\mathbf{v}^{T} \delta \mathbf{d}$ into (24) gives

$$
\delta \mathbf{d}_{\ell}^{i}=\mathbf{Q}^{T} \delta \mathbf{d}^{i}+\mathbf{Q}^{T}\left(\begin{array}{c}
y^{i L} \\
-x^{i L}
\end{array}\right) \mathbf{v}^{T} \delta \mathbf{d}
$$

Next, realizing that $\mathbf{Q}^{T}\left(\begin{array}{c}y^{i L} \\ -x^{i L}\end{array}\right)=\left(\begin{array}{c}y_{\ell}^{i} \\ -x_{\ell}^{i}\end{array}\right),(27)$ becomes

$$
\delta \mathbf{d}_{\ell}^{i}=\mathbf{Q}^{T} \delta \mathbf{d}^{i}+\left(\begin{array}{c}
y_{\ell}^{i} \\
-x_{\ell}^{i}
\end{array}\right) \mathbf{v}^{T} \delta \mathbf{d}
$$

Using (28), an alternative form is written for all neighbors and the current point L as

$$
\delta \mathbf{d}_{\ell}=\left(\overline{\mathbf{Q}}+\overline{\mathbf{x}}_{\ell} \mathbf{v}^{T}\right) \delta \mathbf{d},
$$

where

$$
\overline{\mathbf{Q}}=\left[\begin{array}{cccc}
{\left[\mathbf{Q}^{T}\right]} & \mathbf{0} & \ldots & \mathbf{0} \\
\mathbf{0} & {\left[\mathbf{Q}^{T}\right]} & & \vdots \\
\vdots & & \ddots & \vdots \\
\mathbf{0} & \ldots & \ldots & {\left[\mathbf{Q}^{T}\right]}
\end{array}\right], \quad \mathbf{0}=\left[\begin{array}{cc}
0 & 0 \\
0 & 0
\end{array}\right]
$$

and

$$
\overline{\mathbf{x}}_{\ell}^{T}=\left[\begin{array}{lllllll}
y_{\ell}^{1} & -x_{\ell}^{1} & y_{\ell}^{2} & -x_{\ell}^{2} & \ldots & y_{\ell}^{n} & -x_{\ell}^{n}
\end{array}\right] .
$$

Note that $\overline{\mathbf{Q}}$ is a $2 n$ by $2 n$ matrix. Then, comparing (29) with (13) it is evident that

$$
\mathbf{T}=\overline{\mathbf{Q}}+\overline{\mathbf{x}}_{\ell} \mathbf{v}^{T}
$$

Copyright (C) 2008 John Wiley \& Sons, Ltd.

Int. J. Numer. Meth. Engng 2008; 00:1-38

Prepared using nmeauth.cls 
All that remains to construct the tangent stiffness matrix (see (17)) is the initial stiffness matrix $\mathbf{K}_{t \sigma}$. The initial stiffness matrix arises from (see (16))

$$
\delta \mathbf{T}^{T} \mathbf{q}_{L \ell}=\mathbf{K}_{t \sigma} \delta \mathbf{d}
$$

The variation of $\mathbf{T}^{T}$ is found by representing the first part of (31) as

$$
\delta \mathbf{T}^{T} \mathbf{q}_{L \ell}=\delta \mathbf{T}^{1} \mathbf{q}_{L \ell}^{1}+\delta \mathbf{T}^{2} \mathbf{q}_{L \ell}^{2}+\ldots=\sum_{j=1}^{2 n} \delta \mathbf{T}^{j} \mathbf{q}_{L \ell}^{j},
$$

where $\mathbf{T}^{j}$ is the $j$ th column of $\mathbf{T}^{T}$ and $\mathbf{q}_{L \ell}^{j}$ is the $j$ th component of $\mathbf{q}_{L \ell}$ (which is a scalar). Working now only with the first term in the summation (32) and using the transpose of (30) gives

$$
\delta \mathbf{T}^{1} \mathbf{q}_{L \ell}^{1}=\mathbf{q}_{L \ell}^{1} \delta\left\{\left\{\begin{array}{c}
\mathbf{e}_{1}^{\prime} \\
\mathbf{0} \\
\vdots \\
\mathbf{0}
\end{array}\right\}+y_{\ell}^{1} \mathbf{v}\right\}=\mathbf{q}_{L \ell}^{1} \mathbf{G}^{1} \delta \mathbf{d},
$$

where $\mathbf{0}^{T}=\left[\begin{array}{ll}0 & 0\end{array}\right]$. From (33), $\mathbf{G}^{1} \delta \mathbf{d}$ must be determined. This is given by

$$
\mathbf{G}^{1} \delta \mathbf{d}=\delta\left\{\left\{\begin{array}{c}
\mathbf{e}_{1}^{\prime} \\
\mathbf{0} \\
\vdots \\
\mathbf{0}
\end{array}\right\}+y_{\ell}^{1} \mathbf{v}\right\}=\left\{\begin{array}{c}
\mathbf{e}_{2}^{\prime} \\
\mathbf{0} \\
\vdots \\
\mathbf{0}
\end{array}\right\} \delta \theta+\delta y_{\ell}^{1} \mathbf{v}+y_{\ell}^{1} \delta \mathbf{v} .
$$

Now note that $\delta y_{\ell}^{1}$ comes from (29), i.e.,

$$
\delta y_{\ell}^{1}=\left\{\left[\begin{array}{lllll}
\mathbf{e}_{2}^{\prime T} & \mathbf{0} & \mathbf{0} & \ldots & \mathbf{0}
\end{array}\right]-x_{\ell}^{1} \mathbf{v}^{T}\right\} \delta \mathbf{d} .
$$

To see this, consider for a moment the generic variable $\mathbf{w}$. The variation of this variable in local coordinates is related to the variation of itself in global coordinates as (see (29))

$$
\delta \mathbf{w}_{\ell}=\left(\overline{\mathbf{Q}}+\overline{\mathbf{x}}_{\ell} \mathbf{v}^{T}\right) \delta \mathbf{w}
$$

Copyright (C) 2008 John Wiley \& Sons, Ltd.

Int. J. Numer. Meth. Engng 2008; 00:1-38

Prepared using nmeauth.cls 
where $\mathbf{w}=\mathbf{X}+\mathbf{d}$ and $\mathbf{w}_{\ell}=\mathbf{X}_{\ell}+\mathbf{d}_{\ell}=\mathbf{x}_{\ell}$. Specifically, $\mathbf{w}_{\ell}^{T}=\left\{\begin{array}{ccccc}x_{\ell}^{1} & y_{\ell}^{1} & \ldots & x_{\ell}^{n} & y_{\ell}^{n}\end{array}\right\}$. Then observe that $\delta \mathbf{w}=\delta \mathbf{d}$ since $\delta \mathbf{X}=0$. By taking only the row of (36) associated with $\delta y_{\ell}^{1}$, (35) is obtained.

If the last term of (34) is not included for now, using (26), (34) and (35) yields

$$
\mathbf{G}^{1, a}=\left\{\begin{array}{c}
\mathbf{e}_{2}^{\prime} \\
\mathbf{0} \\
\vdots \\
\mathbf{0}
\end{array}\right\} \mathbf{v}^{T}+\mathbf{v}\left\{\begin{array}{c}
\mathbf{e}_{2}^{\prime} \\
\mathbf{0} \\
\vdots \\
\mathbf{0}
\end{array}\right\}^{T}-x_{\ell}^{1} \mathbf{v} \mathbf{v}^{T},
$$

which is symmetric. In order to obtain the complete form of $\mathbf{G}$ it is necessary to determine $\delta \mathbf{v}$. To this end, from (26) and defining $\mathbf{g}:=\mathbf{a} \ell$,

$$
\mathbf{v}=\frac{1}{a^{2}+b^{2}}(b \mathbf{c}-a \mathbf{g}) .
$$

Then, taking the variation of (37) gives, by use of the product rule,

$$
\delta \mathbf{v}=\delta\left(\frac{1}{a^{2}+b^{2}}\right)(b \mathbf{c}-a \mathbf{g})+\frac{1}{a^{2}+b^{2}} \delta(b \mathbf{c}-a \mathbf{g}) .
$$

Now observe that

$$
\delta\left(\frac{1}{a^{2}+b^{2}}\right)=\delta\left(\left(a^{2}+b^{2}\right)^{-1}\right)=-\left(a^{2}+b^{2}\right)^{-2}\left(2 a \mathbf{c}^{T}+2 b \mathbf{g}^{T}\right) \delta \mathbf{d}=\frac{-2\left(a \mathbf{c}^{T}+b \mathbf{g}^{T}\right)}{\left(a^{2}+b^{2}\right)^{2}} \delta \mathbf{d},
$$

and

$$
\delta(b \mathbf{c}-a \mathbf{g})=\delta b \mathbf{c}-\delta a \mathbf{g}=\left(\mathbf{c g}^{T}-\mathbf{g c}^{T}\right) \delta \mathbf{d} .
$$

Substituting (39) and (40) into (38) yields

$$
\delta \mathbf{v}=\frac{-2\left(a \mathbf{c}^{T}+b \mathbf{g}^{T}\right)}{\left(a^{2}+b^{2}\right)^{2}}(b \mathbf{c}-a \mathbf{g}) \delta \mathbf{d}+\frac{\left(\mathbf{c g}^{T}-\mathbf{g c}^{T}\right)}{a^{2}+b^{2}} \delta \mathbf{d},
$$

which after some algebraic simplifications reduces to

$$
\delta \mathbf{v}=\left[\frac{2 a b\left(\mathbf{g g}^{T}-\mathbf{c c}^{T}\right)+\left(a^{2}-b^{2}\right)\left(\mathbf{c g}^{T}+\mathbf{g c}^{T}\right)}{\left(a^{2}+b^{2}\right)^{2}}\right] \delta \mathbf{d} \equiv \mathbf{V}^{T} \delta \mathbf{d},
$$

Copyright (C) 2008 John Wiley \& Sons, Ltd.

Int. J. Numer. Meth. Engng 2008; 00:1-38

Prepared using nmeauth.cls 
where the matrix $\mathbf{V}$ is symmetric. The expression for $\mathbf{V}^{T}$ in the above equation is identical to that found by Crisfield and Moita [4] with the exception that their denominator is not squared (a likely typographical error). Note also, that the last term of $\mathbf{G}^{1}$, which includes the variation of $\mathbf{v}$, has insignificant effect on convergence and may be neglected (see Crisfield [19] for a discussion on neglecting $\mathbf{v}$ and also the more recent work by Rankin [18]). However, for completeness it is kept here. Hence, having

$$
\mathbf{G}^{1, b}=y_{\ell}^{1} \mathbf{V}^{T}
$$

the final expression for $\mathbf{G}^{1}$ is found as

$$
\mathbf{G}^{1}=\mathbf{G}^{1, a}+\mathbf{G}^{1, b} .
$$

However, the matrix $\mathbf{G}^{1}$ is only sufficient to construct the first term in the summation (32). The other $\mathbf{G}^{j}$ matrices are found similarly. Hence, the initial stiffness matrix is calculated as $\mathbf{K}_{t \sigma}=\sum_{j=1}^{2 n} \mathbf{q}_{L \ell}^{j} \mathbf{G}^{j}$ and subsequently the entire tangent stiffness matrix as given in (17).

As additional information the calculation of $\mathbf{G}^{2}$ is demonstrated next. Starting with the second term in the summation of (32) yields

$$
\delta \mathbf{T}^{2} \mathbf{q}_{L \ell}^{2}=\mathbf{q}_{L \ell}^{2} \delta\left\{\left\{\begin{array}{c}
\mathbf{e}_{2}^{\prime} \\
\mathbf{0} \\
\vdots \\
\mathbf{0}
\end{array}\right\}-x_{\ell}^{1} \mathbf{v}\right\}=\mathbf{q}_{L \ell}^{2} \mathbf{G}^{2} \delta \mathbf{d} .
$$

From (42), $\mathbf{G}^{2} \delta \mathbf{d}$ is determined, which is given by

$$
\mathbf{G}^{2} \delta \mathbf{d}=\delta\left\{\left\{\begin{array}{c}
\mathbf{e}_{2}^{\prime} \\
\mathbf{0} \\
\vdots \\
\mathbf{0}
\end{array}\right\}-x_{\ell}^{1} \mathbf{v}\right\}=\left\{\begin{array}{c}
-\mathbf{e}_{1}^{\prime} \\
\mathbf{0} \\
\vdots \\
\mathbf{0}
\end{array}\right\} \delta \theta+\delta\left(-x_{\ell}^{1}\right) \mathbf{v}+\left(-x_{\ell}^{1}\right) \delta \mathbf{v}
$$

Copyright (C) 2008 John Wiley \& Sons, Ltd.

Int. J. Numer. Meth. Engng 2008; 00:1-38

Prepared using nmeauth.cls 
Now note that $\delta x_{\ell}^{1}$ arises in a similar fashion as that described after (35), i.e.,

$$
\delta x_{\ell}^{1}=\left\{\left[\begin{array}{lllll}
\mathbf{e}_{1}^{\prime T} & \mathbf{0} & \mathbf{0} & \ldots & \mathbf{0}
\end{array}\right]+y_{\ell}^{1} \mathbf{v}^{T}\right\} \delta \mathbf{d} .
$$

Then taking (43) and using (26), (44) and (41) gives

$$
\mathbf{G}^{2}=\left\{\begin{array}{c}
-\mathbf{e}_{1}^{\prime} \\
\mathbf{0} \\
\vdots \\
\mathbf{0}
\end{array}\right\} \mathbf{v}^{T}+\mathbf{v}\left\{\begin{array}{c}
-\mathbf{e}_{1}^{\prime} \\
\mathbf{0} \\
\vdots \\
\mathbf{0}
\end{array}\right\}^{T}-y_{\ell}^{1} \mathbf{v} \mathbf{v}^{T}-x_{\ell}^{1} \mathbf{V}^{T}
$$

Lastly, expressions for the generic cases of $\mathbf{G}^{2 i-1}$ and $\mathbf{G}^{2 i}$ are given below. In general, for $i=1$ to $n$

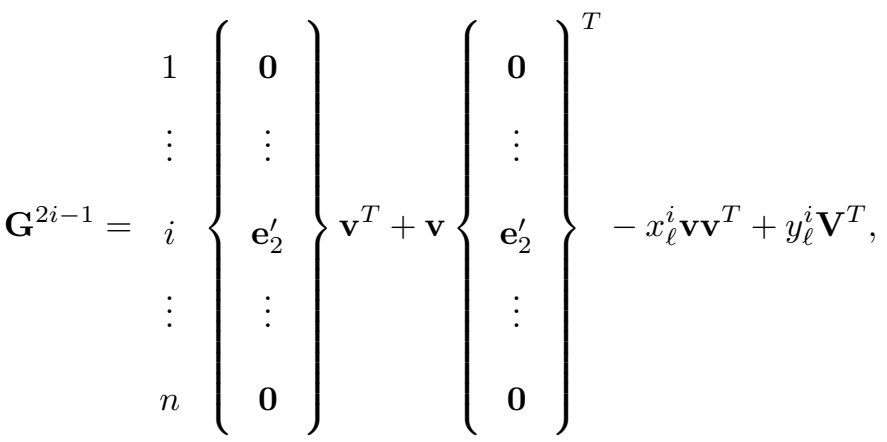

$$
\begin{aligned}
& \mathbf{G}^{2 i}=\left\{\begin{array}{c}
\mathbf{0} \\
\vdots \\
-\mathbf{e}_{1}^{\prime} \\
\vdots \\
\mathbf{0}
\end{array}\right\} \mathbf{v}^{T}+\mathbf{v}\left\{\begin{array}{c}
\mathbf{0} \\
\vdots \\
-\mathbf{e}_{1}^{\prime} \\
\vdots \\
\mathbf{0}
\end{array}\right\}^{T}-y_{\ell}^{i} \mathbf{v} \mathbf{v}^{T}-x_{\ell}^{i} \mathbf{V}^{T}
\end{aligned}
$$

\subsection{Nonlinear material stiffness}

If plasticity is included in the co-rotational formulation then it is necessary to update the material properties during each load step of the analysis. Hence, the local tangent stiffness

Copyright (C) 2008 John Wiley \& Sons, Ltd.

Int. J. Numer. Meth. Engng 2008; 00:1-38

Prepared using nmeauth.cls 
matrix $\mathbf{K}_{t \ell}$ takes the following form:

$$
\mathbf{K}_{t \ell}=\int_{\Omega} \mathbf{B}^{T} \mathbf{C}_{e p} \mathbf{B} d V
$$

where $\mathbf{C}_{e p}$ is the elasto-plastic modulus matrix that evolves during each load step if the local trial stresses fall outside the yield surface such as in a plane stress $J 2$ plasticity formulation with radial return (see Simo and Taylor [20] and Simo and Hughes [21]). All other formulas remain the same.

\subsection{Load control algorithm for a meshfree co-rotational formulation}

An algorithm for the co-rotational formulation in a meshfree setting is given below. The given algorithm is for a linear elastic or elasto-plastic material. In the following, the vectors $\mathbf{d}$ represent meshfree nodal coefficients whereas the vectors $\mathbf{u}$ represent displacements.

1. Set up storage variables

2. Loop over load increments

(a) Create $\Delta \boldsymbol{f}^{n+1}$

(b) Construct $\boldsymbol{f}_{i n t}^{n}$ for each node L and its neighbors based on current stresses, $\boldsymbol{\sigma}^{n}$

(c) Construct $\mathbf{K}^{n}$ based on current $\boldsymbol{f}_{i n t}^{n}$ with current $\Delta \mathbf{d}_{I \ell}$ values and $\mathbf{u}^{n}$

(d) Modify $\Delta \boldsymbol{f}^{n+1}$ and $\mathbf{K}^{n}$ to account for supports

(e) Solve for $\Delta \mathbf{d}_{I}=\left(\mathbf{K}^{n}\right)^{-1} \Delta \boldsymbol{f}^{n+1}$

(f) Calculate displacements $\Delta \mathbf{u}^{n+1}$ based on $\Delta \mathbf{d}_{I}$

(g) Calculate $\Delta \mathbf{d}_{I \ell}$ based on $\mathbf{u}^{n}+\Delta \mathbf{u}^{n+1}$

(h) Calculate the incremental nodal strains based on the latest $\Delta \mathbf{d}_{I \ell}$

(i) Calculate current stresses $\boldsymbol{\sigma}^{n+1}$ (based on elastic or elasto-plastic constitutive relations)

Copyright (C) 2008 John Wiley \& Sons, Ltd.

Int. J. Numer. Meth. Engng 2008; 00:1-38

Prepared using nmeauth.cls 
(j) Construct $\boldsymbol{f}_{\text {int }}^{n+1}$ for each node L and its neighbors based on current stresses, $\boldsymbol{\sigma}^{n+1}$

(k) Update global stiffness to get $\mathbf{K}^{n+1}$ based on current $\boldsymbol{f}_{i n t}^{n+1}$ with current $\Delta \mathbf{d}_{I \ell}$ values and $\mathbf{u}^{n}+\Delta \mathbf{u}^{n+1}$

(l) Modify $\mathbf{K}^{n+1}$ to account for supports

(m) Initialize variables for Newton-Raphson iterations, $k=0$, tol $=10^{-2}$ and maxiter $=100$

(n) Calculate the residual $\mathbf{g}_{(k)}^{n+1}=\boldsymbol{f}_{\text {int }(k)}^{n+1}-\boldsymbol{f}^{n+1}$

(o) Begin Newton-Raphson Iterations, while $\left|\mathbf{g}_{(k)}^{n+1}\right|>$ tol and $k<=$ maxiter

i. $\delta \mathbf{d}_{I}^{(k)}=-\left(\mathbf{K}_{(k)}^{n+1}\right)^{-1} \mathbf{g}_{(k)}^{n+1}$

ii. $\Delta \mathbf{d}_{I}^{(k+1)}=\Delta \mathbf{d}_{I}^{(k)}+\delta \mathbf{d}_{I}^{(k)}$

iii. Calculate displacements $\Delta \mathbf{u}_{(k+1)}^{n+1}$ based on $\Delta \mathbf{d}_{I}^{(k+1)}$

iv. Calculate $\Delta \mathbf{d}_{I \ell}^{(k+1)}$ based on $\mathbf{u}^{n}+\Delta \mathbf{u}_{(k+1)}^{n+1}$

v. Calculate incremental nodal strains based on the latest $\Delta \mathbf{d}_{I \ell}^{(k+1)}$

vi. Calculate current stresses $\boldsymbol{\sigma}_{(k+1)}^{n+1}$

vii. Construct $\boldsymbol{f}_{i n t(k+1)}^{n+1}$ for each node L and its neighbors based on current stresses, $\boldsymbol{\sigma}_{(k+1)}^{n+1}$

viii. Update global stiffness to get $\mathbf{K}_{(k+1)}^{n+1}$ based on current $\boldsymbol{f}_{i n t(k+1)}^{n+1}$ with current $\Delta \mathbf{d}_{I \ell}^{(k+1)}$ values and $\mathbf{u}^{n}+\Delta \mathbf{u}_{(k+1)}^{n+1}$

ix. Modify $\mathbf{K}_{(k+1)}^{n+1}$ to account for supports

x. Calculate the residual $\mathbf{g}_{(k+1)}^{n+1}$

xi. Update iteration variable $k=k+1$

xii. If $k=$ maxiter and $\mathbf{g}_{(k)}^{n+1}>t o l$, provide warning that equilibrium tolerance Copyright (C) 2008 John Wiley \& Sons, Ltd. Int. J. Numer. Meth. Engng 2008; 00:1-38 
not met

(p) End while loop of Newton-Raphson iterations

(q) Update strain $\varepsilon^{n+1}=\varepsilon^{n}+\Delta \varepsilon^{n+1}$

(r) Update displacements $\mathbf{u}^{n+1}=\mathbf{u}^{n}+\Delta \mathbf{u}^{n+1}$

(s) Update stresses

3. End loop over prescribed load increments

\section{MAXIMUM-ENTROPY BASIS FUNCTIONS}

In meshfree Galerkin methods, moving least squares (MLS) approximants [22] and natural neighbor interpolation schemes $[23,24]$ have been widely used, whereas maximum-entropy basis functions are of more recent origin $[25,26]$. For general overviews of meshfree methods and meshfree approximants, the interested reader is referred to Belytschko et al. [27], Li and Liu [28], and Sukumar and Wright [29]. In this paper, maximum-entropy basis functions are used to construct the trial and test approximations that appear in the weak form. Maximumentropy basis functions satisfy a weak Kronecker-delta property on the boundary, which greatly simplifies the imposition of essential boundary conditions [26].

In two dimensions, the constant and linear reproducing conditions, namely $\sum_{a=1}^{n} \phi_{a}(\mathbf{x})=1$, $\sum_{a=1}^{n} \phi_{a}(\mathbf{x}) \mathbf{x}_{a}=\mathbf{x}$, do not prescribe unique basis functions if $n>3$. The Shannon entropy in Reference [25] and a modified entropy functional in Reference [26] are used to regularize the problem to obtain unique basis functions for any $n$. The entropy functional of Arroyo and Ortiz [26] is generalized in Sukumar and Wright [29] on using the notion of a prior within the Copyright (C) 2008 John Wiley \& Sons, Ltd.

Int. J. Numer. Meth. Engng 2008; 00:1-38

Prepared using nmeauth.cls 
Shannon-Jaynes entropy functional.

The variational formulation for maximum-entropy basis functions using the Shannon-Jaynes entropy functional is: find $\phi_{a}(\mathbf{x}) \geq 0$ as the solution of the following constrained optimization problem:

$$
\max _{\phi \in \mathbb{R}_{+}^{n}}-\sum_{a=1}^{n} \phi_{a}(\mathbf{x}) \ln \left(\frac{\phi_{a}(\mathbf{x})}{w_{a}(\mathbf{x})}\right)
$$

subject to the linear reproducing conditions:

$$
\begin{array}{r}
\sum_{a=1}^{n} \phi_{a}(\mathbf{x})=1 \\
\sum_{a=1}^{n} \phi_{a}(\mathbf{x})\left(\mathbf{x}_{a}-\mathbf{x}\right)=\mathbf{0}
\end{array}
$$

where $w_{a}(\mathbf{x})$ is a prior estimate (weight function), and $\mathbb{R}_{+}^{n}$ is the non-negative orthant. The prior weight, $w_{a}(\mathbf{x})$, is the initial estimate of the basis function $\phi_{a}(\mathbf{x})$. If $w_{a}(\mathbf{x})=1$ for all $a$, then the Shannon entropy functional, $-\sum_{a} \phi_{a} \ln \phi_{a}$, is obtained. On using the method of Lagrange multipliers, the solution of the variational problem is [29]:

$$
\phi_{a}(\mathbf{x})=\frac{Z_{a}(\mathbf{x} ; \boldsymbol{\lambda})}{Z(\mathbf{x} ; \boldsymbol{\lambda})}, \quad Z_{a}(\mathbf{x} ; \boldsymbol{\lambda})=w_{a}(\mathbf{x}) \exp \left(-\boldsymbol{\lambda} \cdot \tilde{\mathbf{x}}_{a}\right)
$$

where $\tilde{\mathbf{x}}_{a}=\mathbf{x}_{a}-\mathbf{x}\left(\mathbf{x}, \mathbf{x}_{a} \in \mathbb{R}^{d}\right)$ are shifted nodal coordinates, $\boldsymbol{\lambda}$ are the $d$ Lagrange multipliers associated with the constraints in (46c), and $Z(\mathbf{x})=\sum_{b} Z_{b}(\mathbf{x} ; \boldsymbol{\lambda})$. A Newton method is used to solve the dual optimization problem $(\min \ln Z)$ to obtain $\boldsymbol{\lambda}$; details on the computation of $\phi_{a}$ and $\nabla \phi_{a}$ are provided in References [26] and [29] for a uniform prior and a Gaussian prior, respectively.

The expressions for the derivatives of the maximum-entropy basis functions for any prior weight function are derived. The notations and approach presented in Arroyo and Ortiz [26] are adopted. In what follows, it is assumed that $\boldsymbol{\lambda}$ is the converged solution for the Lagrange Copyright (C) 2008 John Wiley \& Sons, Ltd.

Int. J. Numer. Meth. Engng 2008; 00:1-38

Prepared using nmeauth.cls 
multipliers and $\nabla \phi_{a}$ is the gradient of the basis function. Equation (47) is written as

$$
\phi_{a}(\mathbf{x} ; \boldsymbol{\lambda})=\frac{\exp \left[f_{a}(\mathbf{x} ; \boldsymbol{\lambda})\right]}{\sum_{b=1}^{n} \exp \left[f_{b}(\mathbf{x} ; \boldsymbol{\lambda})\right]}, \quad f_{a}(\mathbf{x} ; \boldsymbol{\lambda})=\ln w_{a}(\mathbf{x})-\boldsymbol{\lambda} \cdot \tilde{\mathbf{x}}_{a},
$$

where $\boldsymbol{\lambda}$ is implicitly dependent on $\mathbf{x}$. Using (48) yields

$$
\nabla \phi_{a}=\phi_{a}\left(\nabla f_{a}-\sum_{b=1}^{n} \phi_{b} \nabla f_{b}\right)
$$

Taking the gradient of $f_{a}$ in (48) and simplifying results in

$$
\nabla f_{a}=\frac{\nabla w_{a}}{w_{a}}+\boldsymbol{\lambda}-\tilde{\mathbf{x}}_{a} \cdot \nabla \boldsymbol{\lambda}
$$

where $\nabla \boldsymbol{\lambda}$ remains to be determined. To this end, on taking the total derivative of both sides of the equality $\mathbf{r}(\mathbf{x} ; \boldsymbol{\lambda})=-\sum_{a=1}^{n} \phi_{a}(\mathbf{x} ; \boldsymbol{\lambda}) \tilde{\mathbf{x}}_{a}=\mathbf{0}$, the following equation is obtained:

$$
D \mathbf{r}=\nabla \mathbf{r}+\nabla_{\lambda} \mathbf{r} \cdot \nabla \boldsymbol{\lambda}=\mathbf{0}
$$

where $\nabla \mathbf{r}$ is the gradient of $\mathbf{r}$ (keeping $\boldsymbol{\lambda}$ fixed) and $\nabla_{\lambda}$ is used to denote the gradient operator with respect to $\lambda$. On using (48) and noting that the Hessian of $\ln Z$ is $\mathbf{H}=\nabla_{\lambda} \mathbf{r}$, the above equation yields

$$
\nabla \boldsymbol{\lambda}=-\mathbf{H}^{-1} \nabla \mathbf{r}=\mathbf{H}^{-1}(\mathbf{A}-\mathbf{I}), \quad \mathbf{H}=\sum_{b=1}^{n} \phi_{b} \tilde{\mathbf{x}}_{b} \otimes \tilde{\mathbf{x}}_{b}, \quad \mathbf{A}=\sum_{b=1}^{n} \phi_{b} \tilde{\mathbf{x}}_{b} \otimes \frac{\nabla w_{b}}{w_{b}},
$$

and therefore $\nabla f_{a}$ in (50) becomes

$$
\nabla f_{a}=\frac{\nabla w_{a}}{w_{a}}+\boldsymbol{\lambda}+\tilde{\mathbf{x}}_{a} \cdot\left[(\mathbf{H})^{-1}-(\mathbf{H})^{-1} \cdot \mathbf{A}\right]
$$

Using the above expression for $\nabla f_{a}$ in (49), the gradient of $\phi_{a}$ is

$$
\nabla \phi_{a}=\phi_{a}\left\{\tilde{\mathbf{x}}_{a} \cdot\left[(\mathbf{H})^{-1}-(\mathbf{H})^{-1} \cdot \mathbf{A}\right]+\frac{\nabla w_{a}}{w_{a}}-\sum_{b=1}^{n} \phi_{b} \frac{\nabla w_{b}}{w_{b}}\right\}
$$

Note that if the prior weight function $w_{a}(\mathbf{x})=\exp \left(-\beta\left|\mathbf{x}_{a}-\mathbf{x}\right|^{2}\right)$ (Gaussian radial basis function), then $\nabla \phi_{a}=\phi_{a} \mathbf{H}^{-1} \cdot \tilde{\mathbf{x}}_{a}$, which appears in the Appendix of Reference [26]. For Copyright (C) 2008 John Wiley \& Sons, Ltd.

Int. J. Numer. Meth. Engng 2008; 00:1-38 Prepared using nmeauth.cls 
the numerical results in this paper, the following quartic prior weight function is used:

$$
w(q)=\left\{\begin{array}{ll}
1-6 q^{2}+8 q^{3}-3 q^{4} & q \leq 1 \\
0 & q>1
\end{array},\right.
$$

where $q=\left\|\mathbf{x}-\mathbf{x}_{a}\right\| / \rho_{a}$ and $\rho_{a}$ is the radius of support for the nodal weight function which is taken as 0.9 times the distance to the fifth nearest neighbor. A software library in Fortran 90 to compute maximum-entropy basis functions is available in the public-domain [30].

The advantages of using maximum-entropy basis functions are revealed in Figure 2. Quartic weight functions, max-ent basis functions and commonly employed moving least squares (MLS) basis functions are depicted on a unit square covered by a $3 \times 3$ nodal grid. For this example, to make the differences between the shape functions visually evident, the support size of the nodal weight function is taken as 1.25 times the distance to the fifth nearest neighbor. It is evident from Fig. $2 \mathrm{f}$ that the interior MLS basis function is not zero on the boundary in contrast to the max-ent basis function (Fig. 2d), which is zero on the boundary of the domain. Furthermore, boundary basis functions using maximum entropy are interpolatory (Fig. 2c), whereas MLS basis functions are not (Fig. 2e). Due to these properties of maximum-entropy basis functions, the imposition of essential boundary conditions in maximum-entropy meshfree methods is performed as in finite element methods.

\subsection{Principle of virtual work and nodal integration}

The weak form (principle of virtual work) for problems in structural mechanics leads to the equilibrium expression

$$
\begin{gathered}
\boldsymbol{f}^{e x t}-\boldsymbol{f}^{i n t}=\mathbf{0} \\
\boldsymbol{f}^{e x t}=\int_{S} \boldsymbol{\phi}^{T} \overline{\boldsymbol{t}} d S, \quad \boldsymbol{f}^{i n t}=\int_{\Omega} \mathbf{B}^{T} \boldsymbol{\sigma} d V
\end{gathered}
$$

Copyright (C) 2008 John Wiley \& Sons, Ltd.

Int. J. Numer. Meth. Engng 2008; 00:1-38

Prepared using nmeauth.cls 


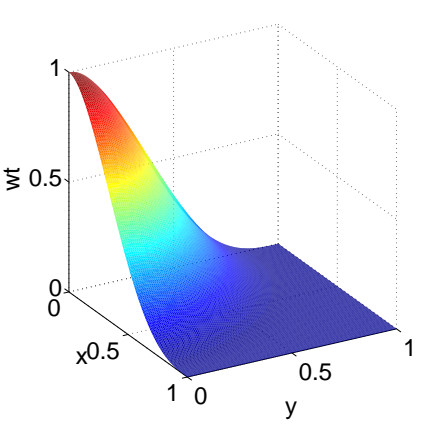

(a)

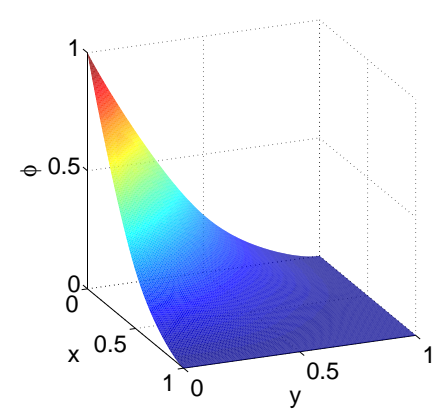

(c)

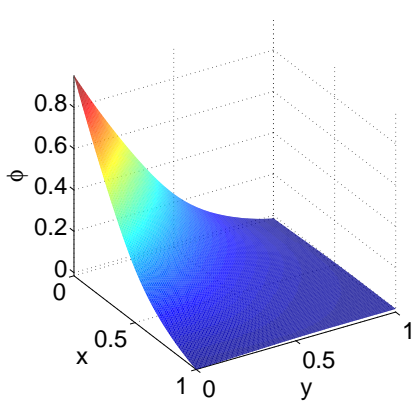

(e)
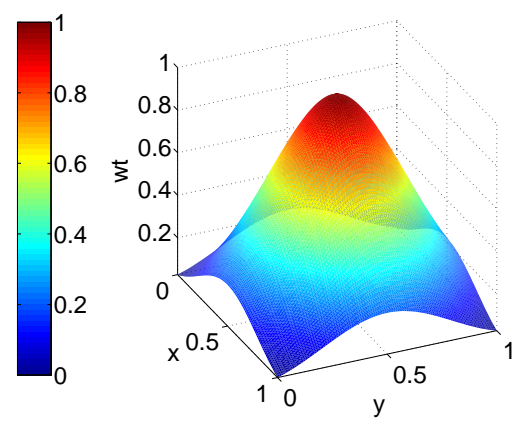

(b)
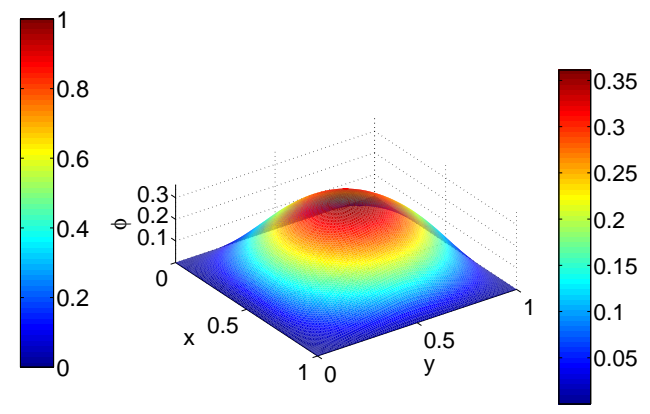

(d)
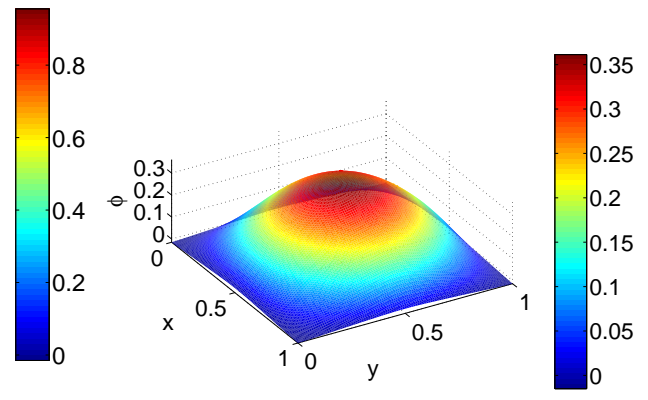

(f)

Figure 2. Max-ent versus MLS basis functions on unit square $(3 \times 3$ grid). Quartic weight function, max-ent and MLS basis functions for corner node in (a),(c),(e) and for center node in (b),(d),(f). 
where $\boldsymbol{\phi}$ is the basis function vector, $\mathbf{B}$ is the local strain-displacement matrix, $\boldsymbol{\sigma}$ is the local Cauchy stress, and $\overline{\boldsymbol{t}}$ is the prescribed traction vector.

In an effort to depart from using elements for the purpose of numerical integration, a nodebased integration technique is used to compute $f^{\text {int }}$ in (54). For node-based integration, a background geometric structure, such as a Voronoi diagram, is still required. This geometric structure is preferable since it is node-based rather than element-based and hence the Jacobian is not needed. A further advantage of nodal integration is that state variables, such as material properties, are associated with nodes rather than elements. The nodal integration procedure adopted here closely follows the integration scheme introduced by Chen et al. [31]. For other forms of nodal integration using stress points, see Duan and Belytschko [32].

Consider the Voronoi cell domain $V_{a}$ and boundary of segments $S_{a}$ enclosing node $a$ as shown in Fig. 3a. Over the domain $V_{a}$, the components of the smoothed strain tensor are

$$
\varepsilon_{i j}\left(\boldsymbol{x}_{a}\right)=\frac{1}{2 A_{a}} \int_{V_{a}}\left(u_{i, j}+u_{j, i}\right) d V=\frac{1}{2 A_{a}} \int_{S_{a}}\left(u_{i} n_{j}+u_{j} n_{i}\right) d S
$$

where $A_{a}$ is the Voronoi cell area associated with node $a$, and $n_{i}$ is the $i$ th component of a unit vector normal to the Voronoi cell boundary $S_{a}$.

Now, similar to FEM, the strain-displacement relation is written as

$$
\varepsilon\left(\boldsymbol{x}_{a}\right)=\sum_{b=1}^{6} \mathbf{B}_{b}\left(\boldsymbol{x}_{a}\right) \mathbf{d}_{b}=\left[\begin{array}{ll}
\mathbf{B}_{1} \mathbf{B}_{2} \cdots \mathbf{B}_{6}
\end{array}\right]\left[\begin{array}{c}
\mathbf{d}_{1} \\
\mathbf{d}_{2} \\
\vdots \\
\mathbf{d}_{6}
\end{array}\right] \equiv \mathbf{B}\left(\boldsymbol{x}_{a}\right) \mathbf{d}
$$

where the index $b$ ranges over the nodes whose associated basis function supports cover any vertex of the Voronoi cell $a$ (i.e., nodes 1 to 6 in Figure 3a). The strain-displacement matrix Copyright (C) 2008 John Wiley \& Sons, Ltd.

Int. J. Numer. Meth. Engng 2008; 00:1-38

Prepared using nmeauth.cls 


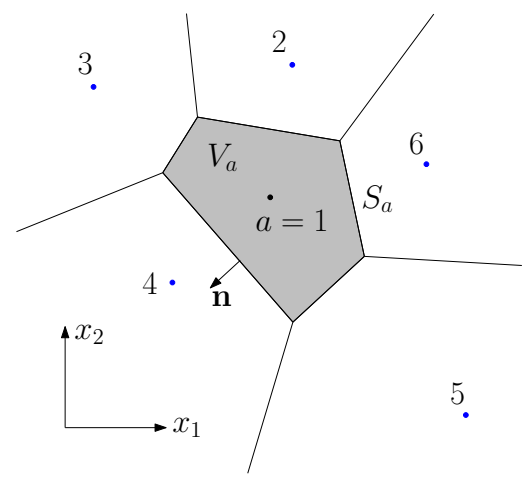

(a)

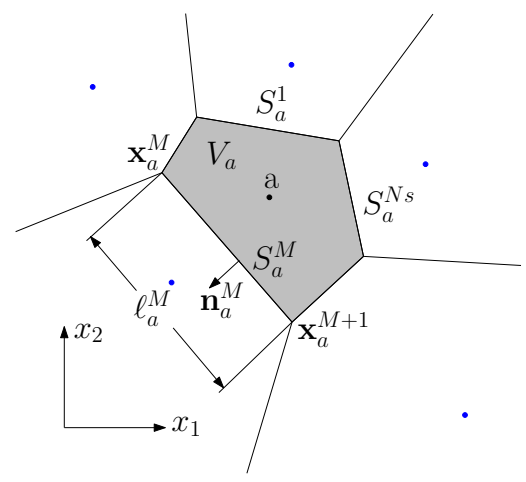

(b)

Figure 3. Nodal integration: (a) Voronoi cell of node $a=1$; and (b) normal and edge definitions.

is:

$$
\mathbf{B}_{b}\left(\boldsymbol{x}_{a}\right)=\left[\begin{array}{cc}
b_{b 1}\left(\boldsymbol{x}_{a}\right) & 0 \\
0 & b_{b 2}\left(\boldsymbol{x}_{a}\right) \\
b_{b 2}\left(\boldsymbol{x}_{a}\right) & b_{b 1}\left(\boldsymbol{x}_{a}\right)
\end{array}\right], \quad b_{b i}\left(\boldsymbol{x}_{a}\right)=\frac{1}{A_{a}} \int_{S_{a}} \phi_{b}(\boldsymbol{x}) n_{i}(\boldsymbol{x}) d S .
$$

To carry out the integration, by numerically evaluating the components of the $\mathbf{B}$ matrix, a two-point trapezoidal rule is employed. As indicated in Figure $3 \mathrm{~b}, \boldsymbol{x}_{a}^{M}$ and $\boldsymbol{x}_{a}^{M+1}$ are the end points of segment $S_{a}^{M}$. The length of the segment is $\ell_{a}^{M}$ and surface normal of the segment is $\boldsymbol{n}_{a}^{M}$. Using these definitions, (57) is rewritten as a summation over the Voronoi cell segments:

$$
b_{b i}\left(\boldsymbol{x}_{a}\right)=\frac{1}{A_{a}} \sum_{M=1}^{N_{s}}\left[\phi_{b}\left(\boldsymbol{x}_{a}^{M}\right) n_{a i}^{M} \frac{\ell_{a}^{M}}{2}+\phi_{b}\left(\boldsymbol{x}_{a}^{M+1}\right) n_{a i}^{M} \frac{\ell_{a}^{M}}{2}\right] .
$$

When the last segment in the summation is reached define $M+1=N_{s}+1 \equiv 1$. Next, noting that (58) only involves evaluation of $\phi_{b} n_{a i}$ at the vertices of the Voronoi cell for node $a$, the following result is obtained:

$$
b_{b i}\left(\boldsymbol{x}_{a}\right)=\frac{1}{A_{a}} \sum_{M=1}^{N_{s}}\left[\frac{1}{2}\left(n_{a i}^{M} \ell_{a}^{M}+n_{a i}^{M+1} \ell_{a}^{M+1}\right) \phi_{b}\left(\boldsymbol{x}_{a}^{M+1}\right)\right] .
$$

Copyright (C) 2008 John Wiley \& Sons, Ltd.

Int. J. Numer. Meth. Engng 2008; 00:1-38

Prepared using nmeauth.cls 
This last equation involves no derivatives of maximum-entropy basis functions. The technique of nodal integration has been used in linear problems [31] and in nonlinear problems with large displacements [33].

On using the strain-displacement relation (56) in (54) gives the local tangent stiffness associated with node $a$ as

$$
\mathbf{K}\left(\boldsymbol{x}_{a}\right)=\mathbf{B}^{T}\left(\boldsymbol{x}_{a}\right) \mathbf{C B}\left(\boldsymbol{x}_{a}\right) A_{a} t .
$$

The thickness of the two-dimensional domain, $t$, is generally taken as unity. Furthermore, $\mathbf{C}$ is the elastic or elasto-plastic modulus matrix depending on the material model in current use. The external force vector $\boldsymbol{f}^{\text {ext }}$ of (54) is found similarly (see Chen et al. [31]).

\subsection{Stabilization of Stiffness Matrix}

Nodal integration instabilities are often manifested by hourglass modes in the calculated deflected shape, by spurious low-energy modes in an eigenanalysis and by locking in near or totally incompressible materials. Hence, some form of stabilization is needed for the stiffness matrix given in (60). Puso et al. [34] proposed the following stabilization scheme:

$$
\mathbf{K}^{s}\left(\boldsymbol{x}_{a}\right)=\mathbf{K}\left(\boldsymbol{x}_{a}\right)+\alpha_{s} \sum_{c \in T_{a}}\left(\mathbf{B}\left(\boldsymbol{x}_{a}\right)-\mathbf{B}^{c}\left(\boldsymbol{x}_{a}\right)\right)^{T} \mathbf{C}_{s}\left(\mathbf{B}\left(\boldsymbol{x}_{a}\right)-\mathbf{B}^{c}\left(\boldsymbol{x}_{a}\right)\right) A_{c} t
$$

where $\mathbf{K}^{s}\left(\boldsymbol{x}_{a}\right)$ is the stabilized matrix, $\alpha_{s}=1.0$ is the stabilization factor and $\mathbf{C}_{s}$ is the stabilization modulus matrix. The first term in the summation of (61) is equivalent to (60) and for each node $a$ the second term is a summation over the set of triangular subcells, $T_{a}$, for Voronoi cell $a$ (see Figure 4). Over each triangular subcell $c$ the $\mathbf{B}^{c}$ matrix is constructed in the same way that $\mathbf{B}$ matrices are constructed over a Voronoi cell.

Consistent with the stabilization scheme explained above, the local internal forces take the Copyright (C) 2008 John Wiley \& Sons, Ltd.

Int. J. Numer. Meth. Engng 2008; 00:1-38

Prepared using nmeauth.cls 


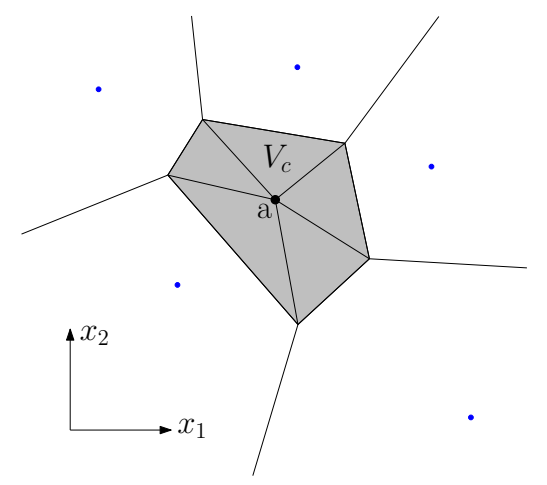

Figure 4. Voronoi cell triangular subcells.

following form:

$$
\mathbf{q}_{L \ell}=\boldsymbol{f}_{\ell}^{i n t}=\int_{\Omega} \mathbf{B}^{T} \boldsymbol{\sigma} d V+\alpha_{s} \sum_{c \in T_{a}}\left[\int_{\Omega}\left(\mathbf{B}-\mathbf{B}^{c}\right)^{T} \mathbf{C}_{s}\left(\mathbf{B}-\mathbf{B}^{c}\right) \mathbf{d}_{\ell} d V_{c}\right]
$$

These local internal forces are transformed to the global level and assembled into a global internal force vector as part of the residual calculation process. The residual is then used in the Newton-Raphson scheme to enforce global equilibrium as indicated in the algorithm of Section 2.5. By use of the consistent internal forces an optimum rate of convergence is maintained in the iterations for global equilibrium.

For elastic materials $\mathbf{C}_{s}=\mathbf{C}_{\text {elast }}$. When constructing $\mathbf{C}_{s}$ for plastic materials with Lamé parameters $\mu$ and $\lambda$, the recommendation of Puso et al. [34] is adopted such that the effective moduli are

$$
\tilde{\mu}=H / 2 \quad \text { and } \quad \tilde{\lambda}=\max (\lambda, 12.5 H)
$$

where $H$ is the linear hardening modulus. The effective elastic modulus $\tilde{E}$ and Poisson's ratio $\tilde{\nu}$ in terms of $\tilde{\mu}$ and $\tilde{\lambda}$ are given by

$$
\tilde{E}=\frac{\tilde{\mu}(3 \tilde{\lambda}+2 \tilde{\mu})}{\tilde{\lambda}+\tilde{\mu}} \quad \text { and } \quad \tilde{\nu}=\frac{\tilde{\lambda}}{2(\tilde{\lambda}+\tilde{\mu})}
$$

Copyright (C) 2008 John Wiley \& Sons, Ltd.

Int. J. Numer. Meth. Engng 2008; 00:1-38

Prepared using nmeauth.cls 


\section{NUMERICAL EXAMPLES}

Numerical results for plane stress are presented using an implicit Newton-Raphson iteration scheme at the global level. At the constitutive level, for inelastic materials, J2 plasticity with an implicit Newton-Raphson iteration scheme using radial return is employed [20].

\subsection{Linear elastic cantilever beam}

A linear elastic cantilever beam with $\nu=0.0, E=100.0 \mathrm{ksi}$ and uniform thickness $t=2.0$ inches is loaded with a uniform load along the vertical free end. Deflected shapes are shown for a regular and irregular grid of nodes in Figures 5a and 5b, respectively. A load displacement plot of a meshfree co-rotational cantilever beam is compared to a 1D co-rotational beam finite element in Figure 5c. The software OpenSees [35] is used to obtain the results for the 1D beam element. The 1D beam element model uses ten beam elements. An analytical solution based on Euler-Bernoulli beam theory with consideration of axial deformations is also shown in the load displacement plot. For both regular and irregular grids, the agreement of the current method with the other solutions is excellent. The final deflected shape of the cantilever corresponds to a load of $10 \mathrm{kips}$, and the plot of stress (Figure 5d) with increasing displacement is shown for model node $A$ indicated. The loading takes the strains of the small strain formulation higher than is recommended ( 25 percent bending strain at node $A$ ); however, the results illustrate robust and smooth results and the effectiveness of the stabilization in suppressing hourglass modes. Figure 5e illustrates the hourglass modes that result when no stabilization is used. In fact, without stabilization, the analysis crashes and fails to even 

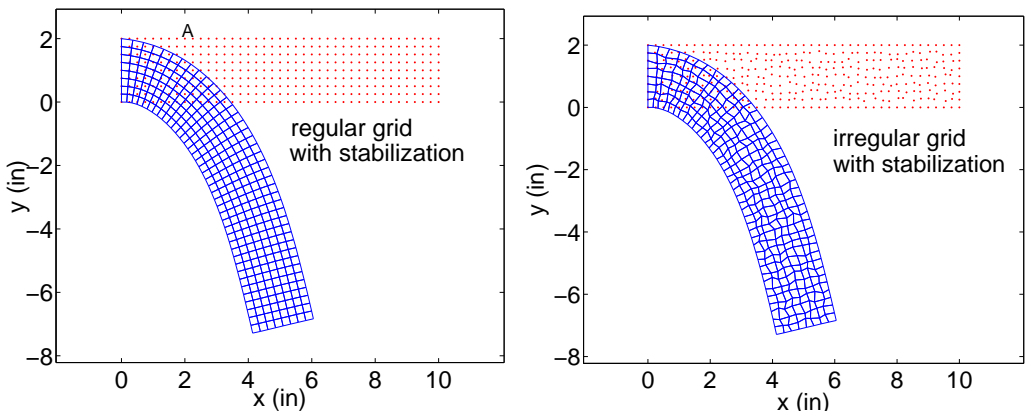

(a)

(b)
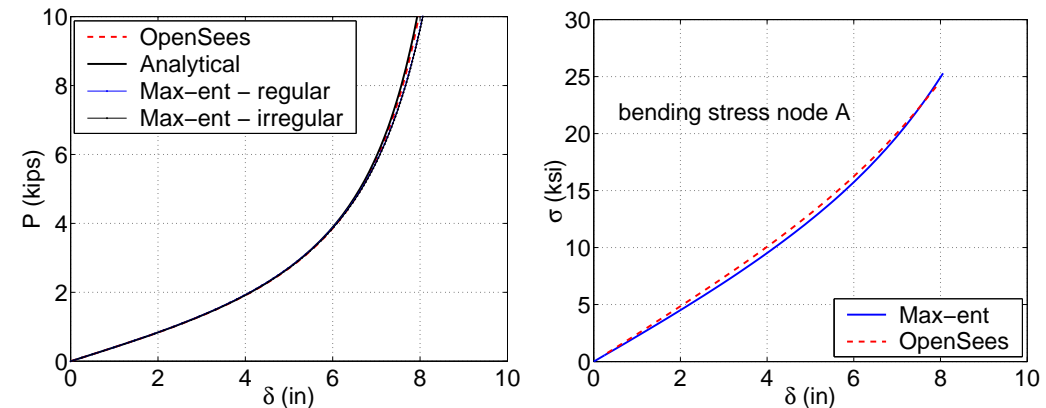

(c)

(d)
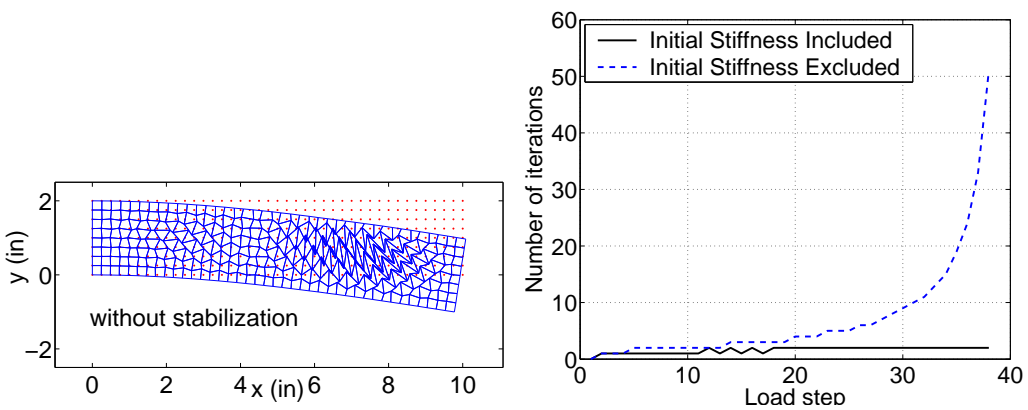

(e)

(f)

Figure 5. 2D meshfree cantilever beam solution compared to 1D co-rotational beam element: (a) final deflected shape (regular grid); (b) final deflected shape (irregular grid); (c) load displacement plot; (d) bending stress; (e) spurious deflected shape without stabilization; and (f) iterations per load step with and without the initial stiffness matrix included.

Copyright (c) 2008 John Wiley \& Sons, Ltd.

Int. J. Numer. Meth. Engng 2008; 00:1-38

Prepared using nmeauth.cls 
converge at loads of about 0.4 kips.

For the above stabilized solution a series of 100 load increments are applied with at most two Newton-Raphson iterations per load step required to reach equilibrium for a residual tolerance of $10^{-2}$. It is noteworthy to point out that in the past some researchers have applied co-rotational formulations without including the variation of the transformation matrix that leads to the initial stiffness matrix. Although for this cantilever beam problem comparable results are obtained for lower load levels by excluding the initial stiffness matrix the number of iterations required for equilibrium increases dramatically. Figure $5 f$ illustrates the number of iterations required without the initial stiffness matrix for the first 38 load steps in an analysis identical to the one described above. The analysis was terminated after the 38 th load step when the number of iterations exceeded 100. This demonstrates the value of a consistent formulation and the loss of the quadratic rate of convergence when the initial stiffness matrix is excluded.

\subsection{Linear elastic circular shallow arch}

A pin supported linear elastic circular shallow arch is loaded with a concentrated force at its central point as shown in Figure 6a. For the arch, $\nu=0.0, E=68.948 \mathrm{kN} / \mathrm{mm}^{2}$, radius is $10581.6 \mathrm{~mm}$, cross-section radial depth is $79.2 \mathrm{~mm}$, and the width of the cross-section is $25.4 \mathrm{~mm}$. The span of the arch from pin to pin is $2540 \mathrm{~mm}$. The arch is modeled with 2761 meshfree nodes, which is similar to 2500 quadrilateral elements. In Figure 6b, the load displacement response, exhibiting snap-through behavior, is compared to results found by using 2500 quadrilateral membrane elements in LS-DYNA [36]. The load displacement results are obtained by using a single node displacement control scheme using 115 displacement increments (see Clarke et al. [37]). The agreement with LS-DYNA is very good. Numerical results are also 


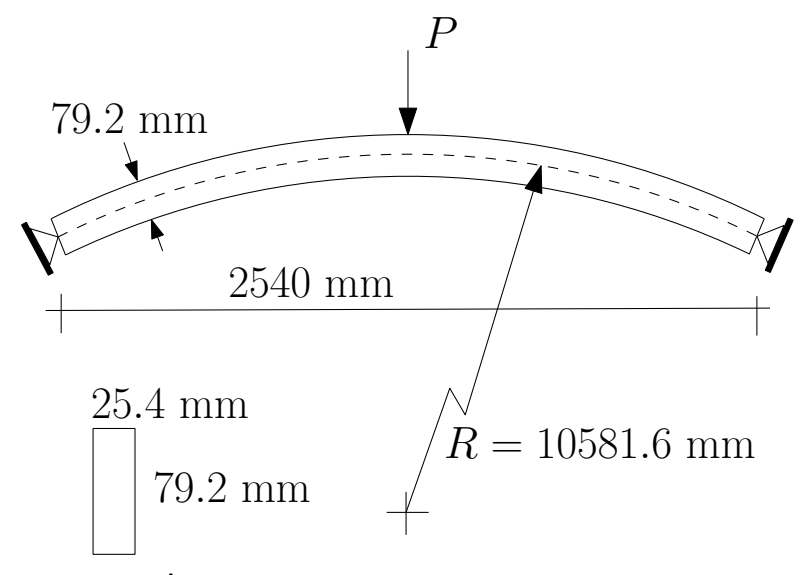

x-section

(a)

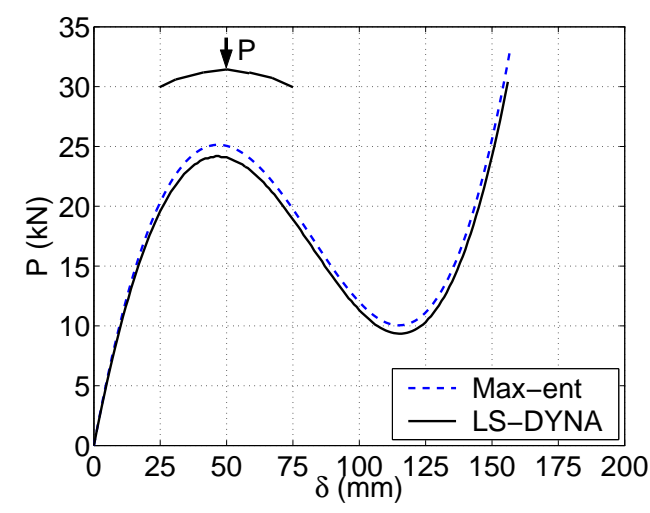

(b)

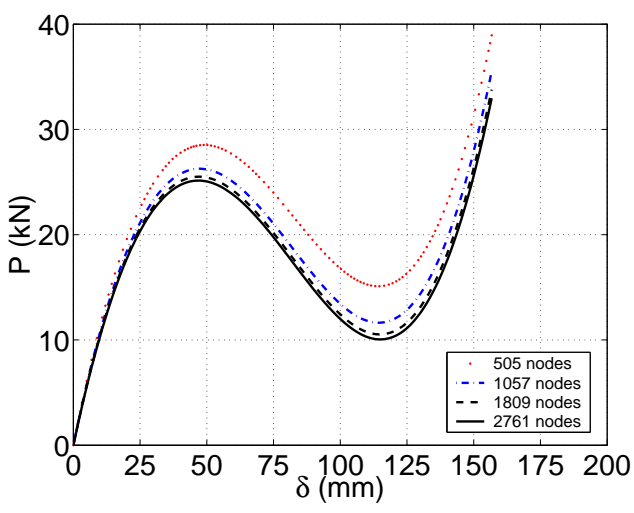

(c)

Figure 6. Results for pin-supported linear elastic circular shallow arch. (a) initial arch configuration;

(b) load displacement plot; (c) max-ent model convergence. 


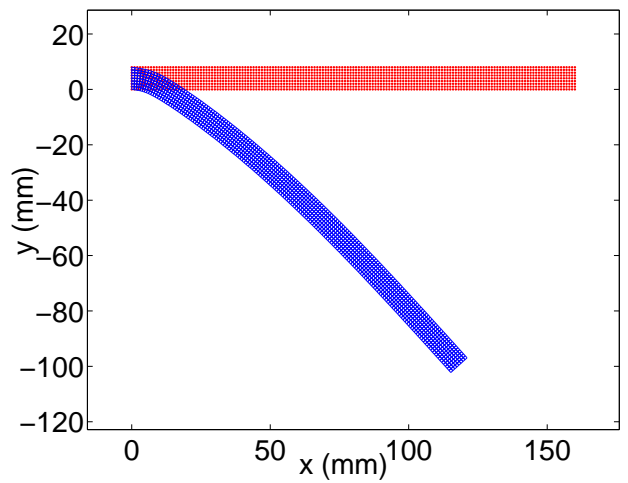

(a)

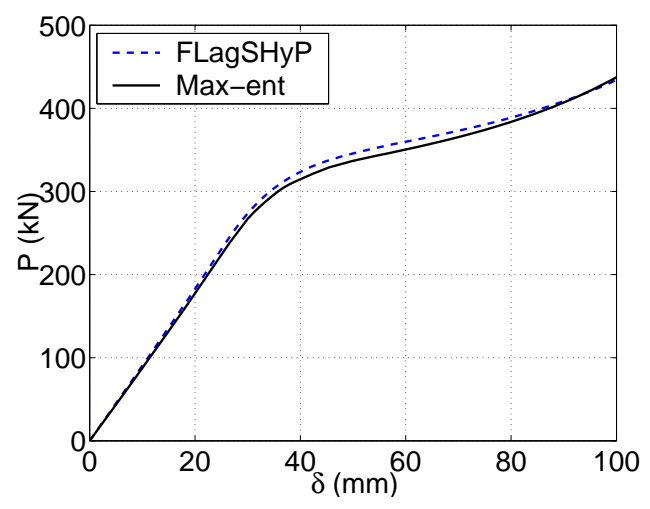

(b)

Figure 7. Results for cantilever with inelastic material behavior included. (a) deflected shape (450 kN load); and (b) load displacement plot.

shown in Figure 6c illustrating the convergence of the meshfree method with grid refinement. The analysis does not correctly capture the snap through behavior when the initial stiffness matrix is excluded, which further illustrates the importance of a variationally consistent corotational formulation.

\subsection{Elasto-plastic cantilever}

As mentioned previously, once a co-rotational formulation is constructed, it is relatively easy to include traditional small strain inelastic material behavior. To demonstrate this, in Figure 7a, a cantilever beam is loaded at its free end with a load of $450 \mathrm{kN}$, which is well beyond first yield. A plastic hinge develops and large rotations of the cantilever beam result. The maximum bending strain is 25 percent. The maximum-entropy model has 1449 nodes. The cantilever is $2 \mathrm{~mm}$ thick, $8 \mathrm{~mm}$ in depth, and is $160 \mathrm{~mm}$ long. A plane stress elasto-plastic material ( $\mathrm{J} 2$ plasticity with 
MESHFREE CO-ROTATIONAL FORMULATION FOR TWO-DIMENSIONAL CONTINUA

radial return [20]) is used with $E=104930 \mathrm{kN} / \mathrm{mm}^{2}, \nu=0.3$, a linear hardening modulus of $284 \mathrm{kN} / \mathrm{mm}^{2}$, and a yield stress of $1550 \mathrm{kN} / \mathrm{mm}^{2}$. Finite element results, obtained by using the finite element large strain hyperelasto-plastic program (FLagShyP) by Bonet and Wood [38], are included for comparison. For the FLagShyP model the same material properties are used, along with 1280 hexahedral elements. Although the FLagShyP model is for a hyperelastoplastic material, for relatively small strains this is comparable to the elasto-plastic material used in the maximum-entropy model. It is evident from the load displacement plot of Figure $7 \mathrm{~b}$ that the finite element and maximum-entropy results are in very good agreement. The analysis is completed by using a displacement control scheme of 42 increments at the free end of the cantilever.

\subsection{Elastic and elasto-plastic T-frame}

A $T$-frame is loaded with a point load as shown in Figure 8a. Figure 8b shows the vertical displacement of node $A$ versus load for elastic and elasto-plastic ( $J 2$ plasticity) materials. The deflected shapes, for the load levels labeled in Figure 8b, are illustrated in Figures 8c-8e. The maximum bending strain is 10 percent and 21 percent for the elastic and elasto-plastic cases, respectively. The results are intended to demonstrate the ability of the co-rotational formulation to capture large displacements and rotations for elastic and elasto-plastic cases. The material properties are as follows: $E=29000 \mathrm{ksi}, \nu=0.3$, linear hardening modulus $H=100 \mathrm{ksi}$ and yield stress $f_{y}=550 \mathrm{ksi}$. The beams and columns of the frame are 4 inch in depth and 1 inch thick. For the elastic case an artificially high yield stress is used so that yielding is avoided during the entire simulation. The analysis is completed using 70 equal (0.3 inch) steps of displacement control at node $A$.

Copyright (C) 2008 John Wiley \& Sons, Ltd.

Int. J. Numer. Meth. Engng 2008; 00:1-38

Prepared using nmeauth.cls 


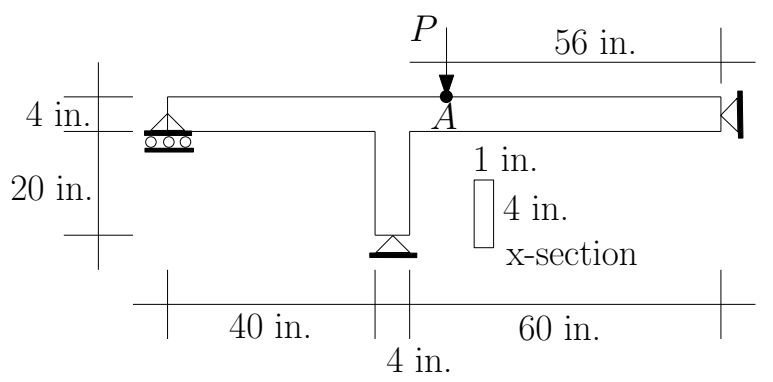

(a)

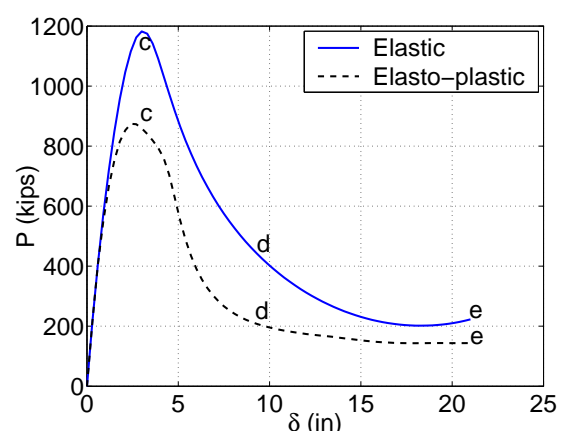

(b)

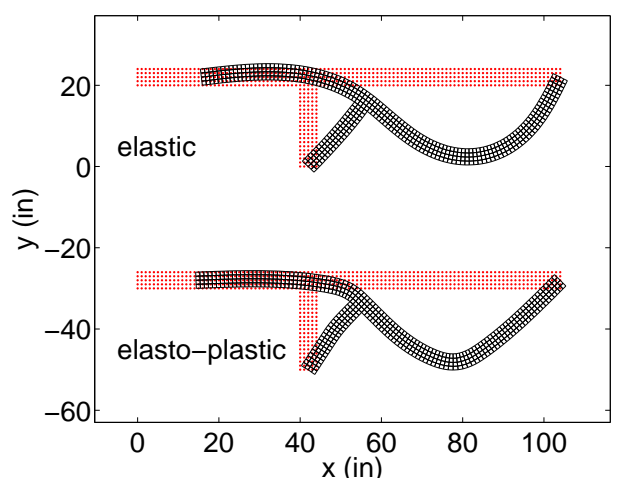

(d)

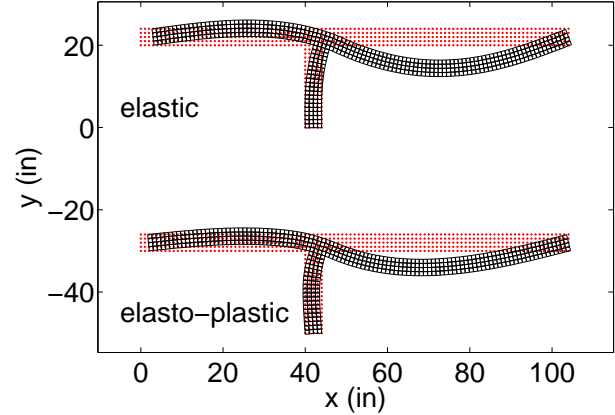

(c)

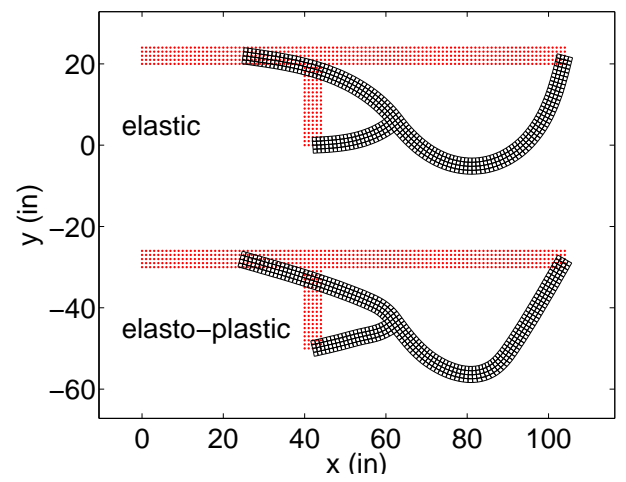

(e)

Figure 8. Results for $T$-frame. (a) initial configuration; (b) load versus displacement for elastic and elasto-plastic cases; and (c),(d),(e) deflected shapes at load levels indicated in (b).

Copyright (C) 2008 John Wiley \& Sons, Ltd.

Int. J. Numer. Meth. Engng 2008; 00:1-38

Prepared using nmeauth.cls 


\section{CONCLUDING REMARKS}

Maximum-entropy basis functions were successfully employed in a meshfree co-rotational formulation for two-dimensional continua. A variationally consistent formulation was required to attain the optimum (quadratic) rate of convergence, and nodal integration and stabilization was applied to representative problems for validation. Benchmark problems such as the cantilever beam, shallow arch, and a $T$-frame were considered with elastic and elasto-plastic material behavior, and the numerical results with the present co-rotational formulation were found to be in good agreement with finite element computations. Notably, the use of stabilization when performing nodal integration prevented the presence of spurious modes in the deflected shape. The numerical results reveal that maximum-entropy basis functions combined with a co-rotational formulation is an effective technique for including large displacements and rotations. This work provides impetus for future research-work on the extensions to finite strains and three-dimensional computations to further the effort to improve large-scale collapse simulations.

\section{ACKNOWLEDGMENTS}

Research support provided by the National Science Foundation, through contract grants CMMI-0826513 and CMMI-0626481, is gratefully acknowledged. The authors thank Yihai Bao for providing the LS-DYNA results for the arch example problem. The comments and suggestions of the anonymous reviewers are also acknowledged.

Copyright (C) 2008 John Wiley \& Sons, Ltd.

Int. J. Numer. Meth. Engng 2008; 00:1-38

Prepared using nmeauth.cls 


\section{REFERENCES}

1. G. Wempner. Finite elements, finite rotations and small strains of flexible shells. International Journal of Solids and Structures, 5:117-153, 1969.

2. T. Belytschko and B. J. Hsieh. Non-linear transient finite element analysis with convected coordinates. International Journal for Numerical Methods in Engineering, 7:255-271, 1973.

3. J. H. Argyris, H. Balmer, J. S. Doltsinis, P.C. Dunne, M. Haase, M. Kleiber, G. A. Malejannakis, H.-P. Mlejenek, M. Müller, and D. W. Scharpf. Finite element method - the natural approach. Computer Methods in Applied Mechanics and Engineering, 17/18:1-106, 1979.

4. M. A. Crisfield and G. F. Moita. A co-rotational formulation for 2-d continua including incompatible modes. International Journal for Numerical Methods in Engineering, 39:2619-2633, 1996.

5. M. A. Crisfield and G. F. Moita. A finite element formulation for 3-d continua using the co-rotational technique. International Journal for Numerical Methods in Engineering, 39:3775-3792, 1996.

6. C. A. Felippa and B. Haugen. A unified formulation of small-strain corotational finite elements: I. Theory. Computer Methods in Applied Mechanics and Engineering, 194:2285-2336, 2005.

7. D. Isobe and M. Tsuda. Seismic collapse analysis of reinforced concrete framed structures using the finite element method. Earthquake Engineering and Structural Dynamics, 32(13):2027-2046, 2003.

8. B.M. Luccioni, R. D. Ambrosini, and R. F. Danesi. Analysis of building collapse under blast loads. Engineering Structures, 26:63-71, 2004.

9. E. Hansen, H. Levine, D. Lawyer, and D. Tennant. Computational failure analysis of reinforced concrete structures subjected to blast loading. In 17th Analysis and Computation Specialty Conference, pages 1-13, St. Louis, Missouri, 2006. ASCE.

10. M. A. M. Torkamani and M. Sonmez. Inelastic large deflection modeling of beam-columns. ASCE Journal of Structural Engineering, 127(8):876-887, 2001.

11. F. F. Taucer, E. Spacone, and G. L. Fenves. A fiber beam-column element for seismic response analysis of reinforced concrete structures. UCB/EERC-91/17, Earthquake Engineering Research Center, University of California, Berkeley, 1991.

12. M. H. Scott and G. L. Fenves. Plastic hinge integration methods for force-based beam-column elements. ASCE Journal of Structural Engineering, 132(2):244-252, 2005.

13. K. Mattiasson, A. Bengtsson, and A. Samuelsson. On the accuracy and efficiency of numerical algorithms for geometrically nonlinear structural analysis. In P. G. Bergan, K. J. Bathe, and W. Wunderlich, editors,

Copyright (C) 2008 John Wiley \& Sons, Ltd.

Int. J. Numer. Meth. Engng 2008; 00:1-38

Prepared using nmeauth.cls 
Finite Element Methods for Nonlinear Problems. Springer Verlag, 1986.

14. T. Belytschko and L. P. Bindeman. Assumed strain stabilization of the eight node hexahedral element. Computer Methods in Applied Mechanics and Engineering, 105:225-260, 1993.

15. A. Masud, C. L. Tham, and W. K. Liu. A stabilized 3-d co-rotational formulation for geometrically nonlinear analysis of multi-layered composite shells. Computational Mechanics, 26:1-12, 2000.

16. W. K. Liu, Y. Guo, S. Tang, and T. Belytschko. A multiple-quadrature eight-node hexahedral finite element for large deformation elastoplastic analysis. Computer Methods in Applied Mechanics and Engineering, 154:69-132, 1998.

17. P. H. Jetteur and S. Cescotto. A mixed finite element for the analysis of large ineleastic strains. International Journal for Numerical Methods in Engineering, 31:229-239, 1991.

18. C. Rankin. Application of linear finite elements to finite strain using corotation. In AIAA Paper \#AIAA2006-1751, 47thAIAA/ASME/ASCE/ASC Structures, Structural Dynamics, and Materials Conference, 2006.

19. M. A. Crisfield. Non-linear Finite Element Analysis of Solids and Structures - Vol 2. John Wiley \& Sons Ltd., Chichester, England, 1997.

20. J. C. Simo and R. L. Taylor. Return mapping algorithm for plane stress elastoplasticity. International Journal for Numerical Methods in Engineering, 22:649-670, 1986.

21. J. C. Simo and T. J. R. Hughes. Computational Inelasticity. Springer-Verlag, New York, 1998.

22. P. Lancaster and K. Salkauskas. Surfaces generated by moving least squares methods. Mathematics of Computation, 37:141-158, 1981.

23. R. Sibson. A vector identity for the Dirichlet tesselation. Mathematical Proceedings of the Cambridge Philosophical Society, 87:151-155, 1980.

24. N. H. Christ, R. Friedberg, and T. D. Lee. Weights of links and plaquettes in a random lattice. Nuclear Physics B, 210(3):337-346, 1982.

25. N. Sukumar. Construction of polygonal interpolants: A maximum entropy approach. International Journal for Numerical Methods in Engineering, 61(12):2159-2181, 2004.

26. M. Arroyo and M. Ortiz. Local maximum-entropy approximation schemes: a seamless bridge between finite elements and meshfree methods. International Journal for Numerical Methods in Engineering, 65(13):2167-2202, 2006.

27. T. Belytschko, Y. Krongauz, D. Organ, M. Fleming, and P. Krysl. Meshless methods: An overview and recent developments. Computer Methods in Applied Mechanics and Engineering, 139:3-47, 1996.

Copyright (C) 2008 John Wiley \& Sons, Ltd.

Int. J. Numer. Meth. Engng 2008; 00:1-38

Prepared using nmeauth.cls 
28. S. Li and W. K. Liu. Meshfree and particle methods and their applications. Applied Mechanics Review, 55(1):1-34, 2002.

29. N. Sukumar and R. W. Wright. Overview and construction of meshfree basis functions: From moving least squares to entropy approximants. International Journal for Numerical Methods in Engineering, 70(2):181-205, 2007.

30. N. Sukumar. Fortran 90 Library for Maximum-Entropy Basis Functions. User's Reference Manual Version 1.4. Code available at http://www.imechanica.org/node/3424, June 2008.

31. J. S. Chen, C. T. Wu, S. Yoon, and Y. You. A stabilized conforming nodal integration for Galerkin meshfree methods. International Journal for Numerical Methods in Engineering, 50:435-466, 2001.

32. Q. Duan and T. Belytschko. Gradient and dilatational stabilizations for stress-point integration in the element-free Galerkin method. International Journal for Numerical Methods in Engineering, 6(5):776$798,2009$.

33. J. S. Chen, S. Yoon, and C. T. Wu. Non-linear version of stabilized conforming nodal integration for Galerkin mesh-free methods. International Journal for Numerical Methods in Engineering, 53:2587-2615, 2002.

34. M. A. Puso, J. S. Chen, E. Zywicz, and W. Elmer. Meshfree and finite element nodal integration methods. International Journal for Numerical Methods in Engineering, 74:416-446, 2008.

35. S. Mazzoni, F. McKenna, M. H. Scott, and G. L. Fenves. OpenSees Command Language Manual. The Regents of the University of California, http://opensees.berkeley.edu/index.php, 2007.

36. J. O. Hallquist. LS-DYNA Theory Manual. Livermore Software Technology Corporation, Livermore, California, 2006.

37. M. J. Clarke and G. J. Hancock. A study of incremental-iterative strategies for non-linear analyses. International Journal for Numerical Methods in Engineering, 29:1365-1391, 1990.

38. J. Bonet and R. D. Wood. Nonlinear Continuum Mechanics for Finite Element Analysis. Cambridge University Press, Cambridge, UK, 2nd edition, 2008.

Copyright (C) 2008 John Wiley \& Sons, Ltd.

Int. J. Numer. Meth. Engng 2008; 00:1-38

Prepared using nmeauth.cls 